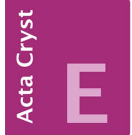

ISSN 2056-9890

\section{Crystal structure of poly[[[ $\mu_{4}-5-(9 H-$ carbazol-9-yl)isophthalato] $\left[\mu_{3}-5-(9 H-\right.$ carbazol-9-yl)isophthalato]bis(dimethyl- formamide)(methanol)dizinc] dimethyl- formamide monosolvate]}

\author{
Liubov M. Lifshits, ${ }^{a}$ Charles Campana ${ }^{\mathrm{b}}$ and Jeremy K. \\ Klosterman ${ }^{\mathrm{a} *}$
}

${ }^{\mathrm{a}}$ Department of Chemistry and Center for Photochemical Sciences, Bowling Green State University, Bowling Green, OH 43403, USA, and ${ }^{\mathbf{b}}$ Bruker AXS Inc., Madison, Wisconsin, USA. *Correspondence e-mail: jkloster@bgsu.edu

Received 26 June 2015; accepted 22 July 2015

Edited by M. Weil, Vienna University of Technology, Austria

The structure of the polymeric title compound, $\left\{\left[\mathrm{Zn}_{2^{-}}\right.\right.$ $\left.\left.\left(\mathrm{C}_{20} \mathrm{H}_{11} \mathrm{NO}_{4}\right)_{2}\left(\mathrm{C}_{3} \mathrm{H}_{7} \mathrm{NO}\right)_{2}\left(\mathrm{CH}_{3} \mathrm{OH}\right)\right] \cdot \mathrm{C}_{3} \mathrm{H}_{7} \mathrm{NO}\right\}_{n}$, comprises carbazolylisophthalate moieties connecting dimetallic tetracarboxylate zinc secondary building units (SBUs) parallel to [100] and [010], leading to a layer-like arrangement parallel to (001). Each SBU consists of two $\mathrm{Zn}$ atoms in slightly distorted tetrahedral and octahedral coordination environments $[\mathrm{Zn} \cdots \mathrm{Zn}=3.5953(6) \AA]$. Three carboxylate groups bridge the two $\mathrm{Zn}$ atoms in a $\mu_{2}-O: O^{\prime}$ mode, whereas the fourth coordinates through a single carboxylate $\mathrm{O}$ atom $\left(\mu_{1}-O\right)$. The $\mathrm{O}$ atoms of two dimethylformamide (DMF) and one methanol molecule complete the $\mathrm{Zn}$ coordination spheres. The methanol ligand interacts with the noncoordinating DMF molecule via an $\mathrm{O}-\mathrm{H} \cdots \mathrm{O}$ hydrogen bond of medium strength. Carbazoles between the layers interdigitate through weak $\mathrm{C}-\mathrm{H} \cdots \pi$ interactions to form a laminar solid stacked along [010]. Two kinds of $\mathrm{C}-\mathrm{H} \cdots \pi$ interactions are present, both with a distance of $2.64 \AA$, between the $\mathrm{H}$ atoms and the centroids, and a third $\mathrm{C}-\mathrm{H} \cdots \pi$ interaction, where the aromatic $\mathrm{H}$ atom is located above the carbazole $\mathrm{N}$-atom lone pair $(\mathrm{H} \cdots \mathrm{N}=2.89 \AA)$. Several $\mathrm{C}-\mathrm{H} \cdots \mathrm{O}$ interactions occur between the coordinating DMF molecule, the DMF solvent molecule, and ligating carboxylate $\mathrm{O}$ atoms.

Keywords: crystal structure; zinc; metal-organic framework; laminar solids.

CCDC reference: 1408495

\section{Related literature}

For solid-state emission behavior and intermolecular packing interactions for a closely related compound where methanol is replaced by ethanol, see: Lifshits et al. (2015). This compound and the title compound are solvatomorphs with identical space groups and comparable lattice parameters. Except for the identity of the coordinating solvent, viz. $\mathrm{MeOH}$ versus $\mathrm{EtOH}$, the other structural components are the same.

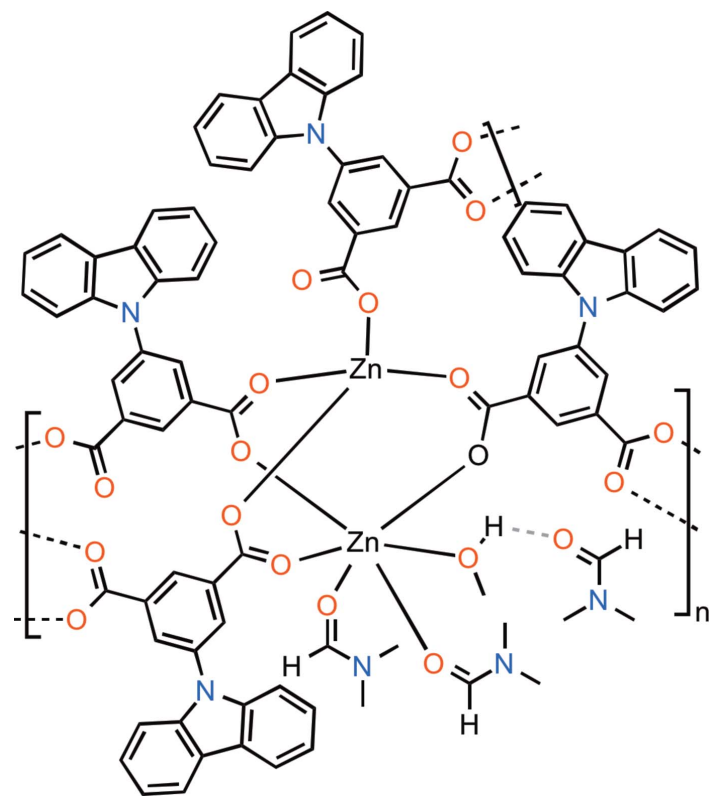

\section{Experimental}

\subsection{Crystal data}

$\left[\mathrm{Zn}_{2}\left(\mathrm{C}_{20} \mathrm{H}_{11} \mathrm{NO}_{4}\right)_{2}\left(\mathrm{C}_{3} \mathrm{H}_{7} \mathrm{NO}\right)_{2^{-}}\right.$ $\left.\left(\mathrm{CH}_{3} \mathrm{OH}\right)\right] \cdot \mathrm{C}_{3} \mathrm{H}_{7} \mathrm{NO}$

Orthorhombic, $P 2_{1} 2_{1} 2_{1}$

$a=10.2867$ (4) §

$b=17.0328(7) \AA$

$c=26.8808(11) \AA$

\subsection{Data collection}

Bruker D8 Venture CMOS diffractometer

Absorption correction: multi-scan (SADABS; Bruker, 2015)

$T_{\min }=0.53, T_{\max }=0.68$

\subsection{Refinement}

$R\left[F^{2}>2 \sigma\left(F^{2}\right)\right]=0.019$

$w R\left(F^{2}\right)=0.045$

$S=1.08$

8611 reflections

629 parameters

546 restraints

$\mathrm{H}$-atom parameters constrained

$\Delta \rho_{\max }=0.41 \mathrm{e}^{-3}$
$M_{r}=1040.66$
$V=4709.8(3) \AA^{3}$

$Z=4$

$\mathrm{Cu} K \alpha$ radiation

$\mu=1.84 \mathrm{~mm}^{-1}$

$T=100 \mathrm{~K}$

$0.40 \times 0.22 \times 0.22 \mathrm{~mm}$

41928 measured reflections 8611 independent reflections 8561 reflections with $I>2 \sigma(I)$ $R_{\text {int }}=0.019$

\footnotetext{
$\Delta \rho_{\text {min }}=-0.18$ e $\AA^{-3}$

Absolute structure: Flack $x$ determined using 3716 quotients $\left[\left(I^{+}\right)-\left(I^{-}\right)\right] /\left[\left(I^{+}\right)+\left(I^{-}\right)\right]$ (Parsons et al, 2013)

Absolute structure parameter: $0.005(3)$
} 
Table 1

Hydrogen-bond geometry $\left(\AA{ }^{\circ}\right)$.

$C g 1, C g 3, C g 4$ and $C g 6$ are the centroids of the $\mathrm{N} 1 / \mathrm{C} 1 / \mathrm{C} 6 / \mathrm{C} 7 / \mathrm{C} 12, \mathrm{C} 1-\mathrm{C} 6, \mathrm{C} 7-$ $\mathrm{C} 12$ and $\mathrm{C} 21-\mathrm{C} 26$ rings, respectively.

\begin{tabular}{|c|c|c|c|c|}
\hline$D-\mathrm{H} \cdots A$ & $D-\mathrm{H}$ & $\mathrm{H} \cdots A$ & $D \cdots A$ & $D-\mathrm{H} \cdots A$ \\
\hline $\mathrm{O} 12-\mathrm{H} 12 \mathrm{O} \cdots \mathrm{O} 11$ & 0.76 & 1.93 & $2.696(2)$ & 174 \\
\hline $\mathrm{C} 42-\mathrm{H} 42 B \cdots \mathrm{O} 11^{\mathrm{i}}$ & 0.98 & 2.52 & $3.327(3)$ & 139 \\
\hline $\mathrm{C} 43-\mathrm{H} 43 \cdots \mathrm{O} 10$ & 0.95 & 2.44 & $3.028(3)$ & 120 \\
\hline $\mathrm{C} 46-\mathrm{H} 46 \cdots \mathrm{O} 5$ & 0.95 & 2.39 & $2.995(2)$ & 121 \\
\hline $\mathrm{C} 47-\mathrm{H} 47 B \cdots \mathrm{O} 3^{\mathrm{ii}}$ & 0.98 & 2.61 & $3.417(3)$ & 140 \\
\hline $\mathrm{C} 47-\mathrm{H} 47 \mathrm{C} \cdots \mathrm{O} 9^{\mathrm{iii}}$ & 0.98 & 2.65 & $3.608(3)$ & 165 \\
\hline $\mathrm{C} 2-\mathrm{H} 2 \cdots C g 6^{\mathrm{iv}}$ & 0.95 & 2.64 & $3.517(2)$ & 153 \\
\hline $\mathrm{C} 10-\mathrm{H} 10 \cdots C g 3^{\mathrm{v}}$ & 0.95 & 2.64 & $3.418(2)$ & 139 \\
\hline $\mathrm{C} 44-\mathrm{H} 44 B \cdots C g 4^{\mathrm{vi}}$ & 0.98 & 2.91 & $3.596(2)$ & 128 \\
\hline $\mathrm{C} 45-\mathrm{H} 45 B \cdots C g 1^{\mathrm{vi}}$ & 0.98 & 2.85 & $3.411(2)$ & 117 \\
\hline
\end{tabular}

Data collection: APEX3 (Bruker, 2015); cell refinement: SAINT (Bruker, 2015); data reduction: $S A I N T$; program(s) used to solve structure: SHELXS97 (Sheldrick, 2008); program(s) used to refine structure: SHELXL2014 (Sheldrick, 2015); molecular graphics:
ShelXle (Hübschle et al., 2011); software used to prepare material for publication: APEX3.

\section{Acknowledgements}

This work was supported by Bowling Green State University and the BGSU Building Strength program.

Supporting information for this paper is available from the $\mathrm{IUCr}$ electronic archives (Reference: WM5180).

\section{References}

Bruker (2015). APEX3, SAINT and SADABS. Bruker AXS Inc., Madison, Wisconsin, USA.

Hübschle, C. B., Sheldrick, G. M. \& Dittrich, B. (2011). J. Appl. Cryst. 44, 12811284.

Lifshits, L. M., Noll, B. C. \& Klosterman, J. K. (2015). Chem. Commun. 51, 11603-11606.

Parsons, S., Flack, H. D. \& Wagner, T. (2013). Acta Cryst. B69, 249-259.

Sheldrick, G. M. (2008). Acta Cryst. A64, 112-122.

Sheldrick, G. M. (2015). Acta Cryst. C71, 3-8. 


\section{supporting information}

Acta Cryst. (2015). E71, m152-m153 [https://doi.org/10.1107/S2056989015013961]

Crystal structure of poly[[[ $\mu_{4}-5-(9 H-c a r b a z o l-9-y l)$ isophthalato] $\left[\mu_{3}-5-(9 H-\right.$ carbazol-9-yl)isophthalato]bis(dimethylformamide)(methanol)dizinc] dimethylformamide monosolvate]

Liubov M. Lifshits, Charles Campana and Jeremy K. Klosterman

S1. Synthesis and crystallization

The title compound was prepared in an analogous manner to $\mathrm{Zn} \mathrm{Cbz} \mathrm{-} \mathrm{EtOH} \mathrm{(Lifshits} \mathrm{et} \mathrm{al.,} \mathrm{2015).} \mathrm{Single} \mathrm{crystals} \mathrm{were}$ obtained by the solvothermal reaction of 5-( $9 H$-carbazol-9-yl)-isophthalic acid and zinc nitrate in DMF/MeOH at room temperature overnight.

\section{S2. Refinement}

All H-atom positions were located from difference maps, then refined with isotropic temperature factors. Subsequently, positional restraints were added for all hydrogen atoms and $U_{\text {iso }}$ values for hydrogen atoms were restrained to $1.2 U_{\text {eq }}$ of the attached atom. 


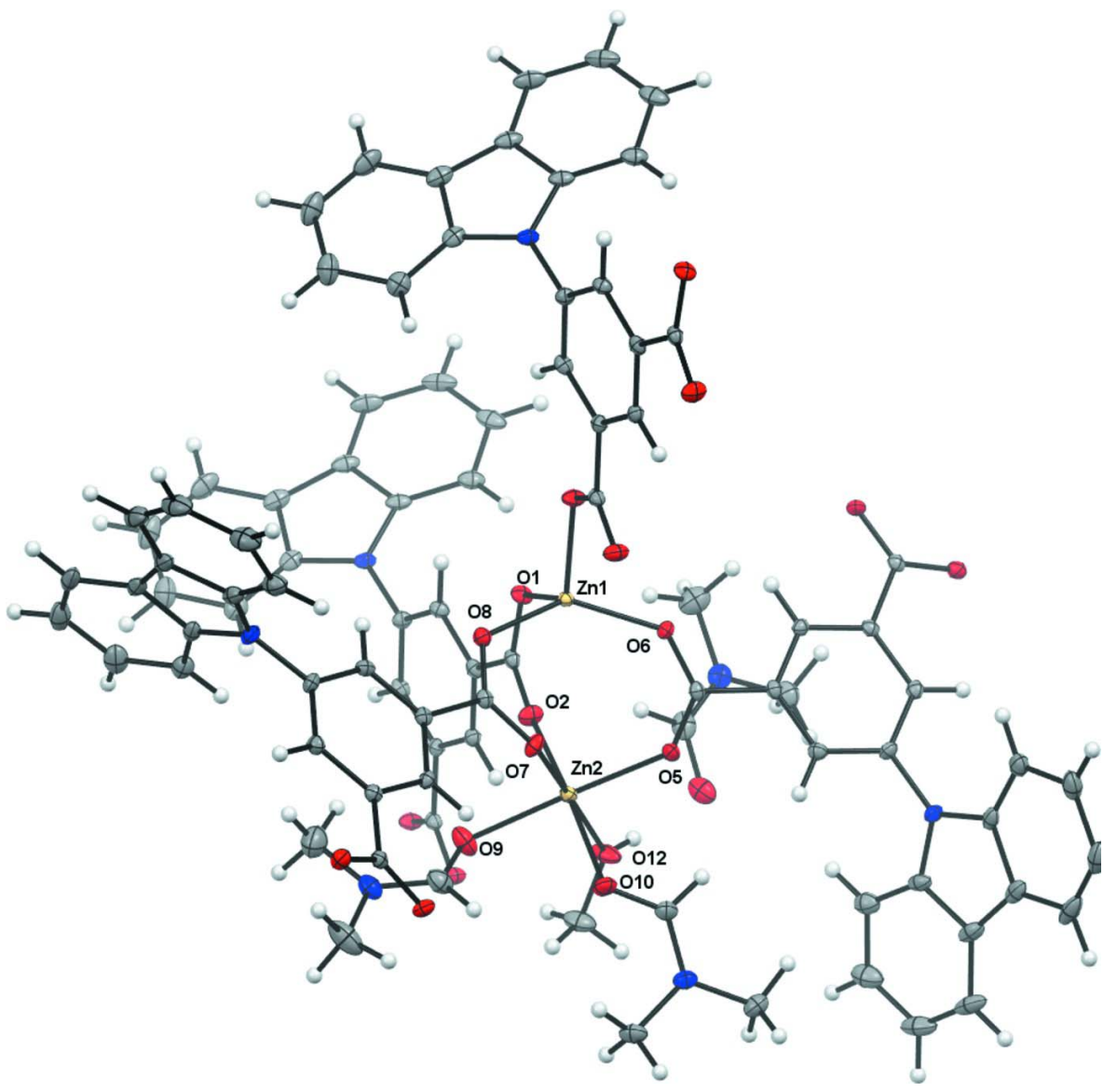

Figure 1

Part of the title structure showing the dimetallic Zn secondary building unit with displacement ellipsoids drawn at the $50 \%$ probability level. 


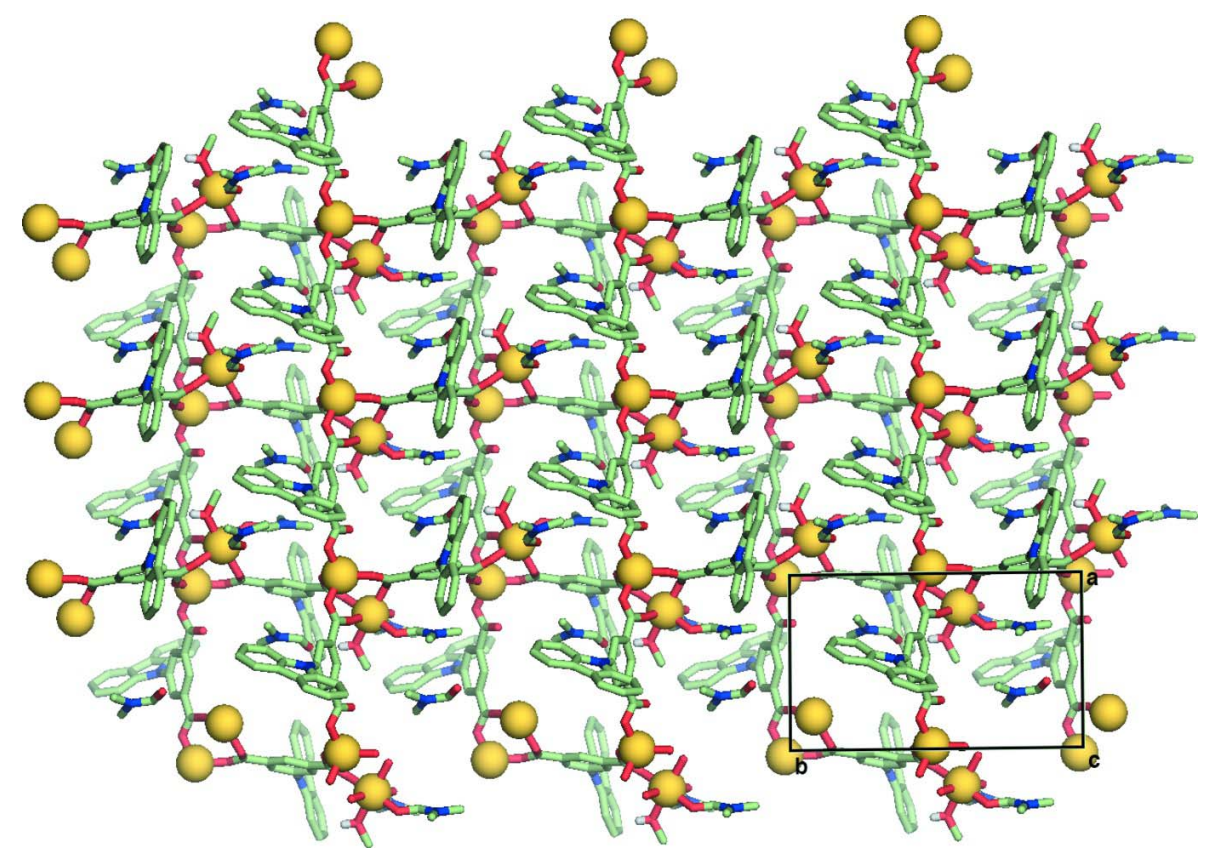

Figure 2

Crystal structure of the title complex in a projection down [001]. Colour code: light green: C, dark blue: N, red: O, white: H, yellow spheres: $\mathrm{Zn}$.

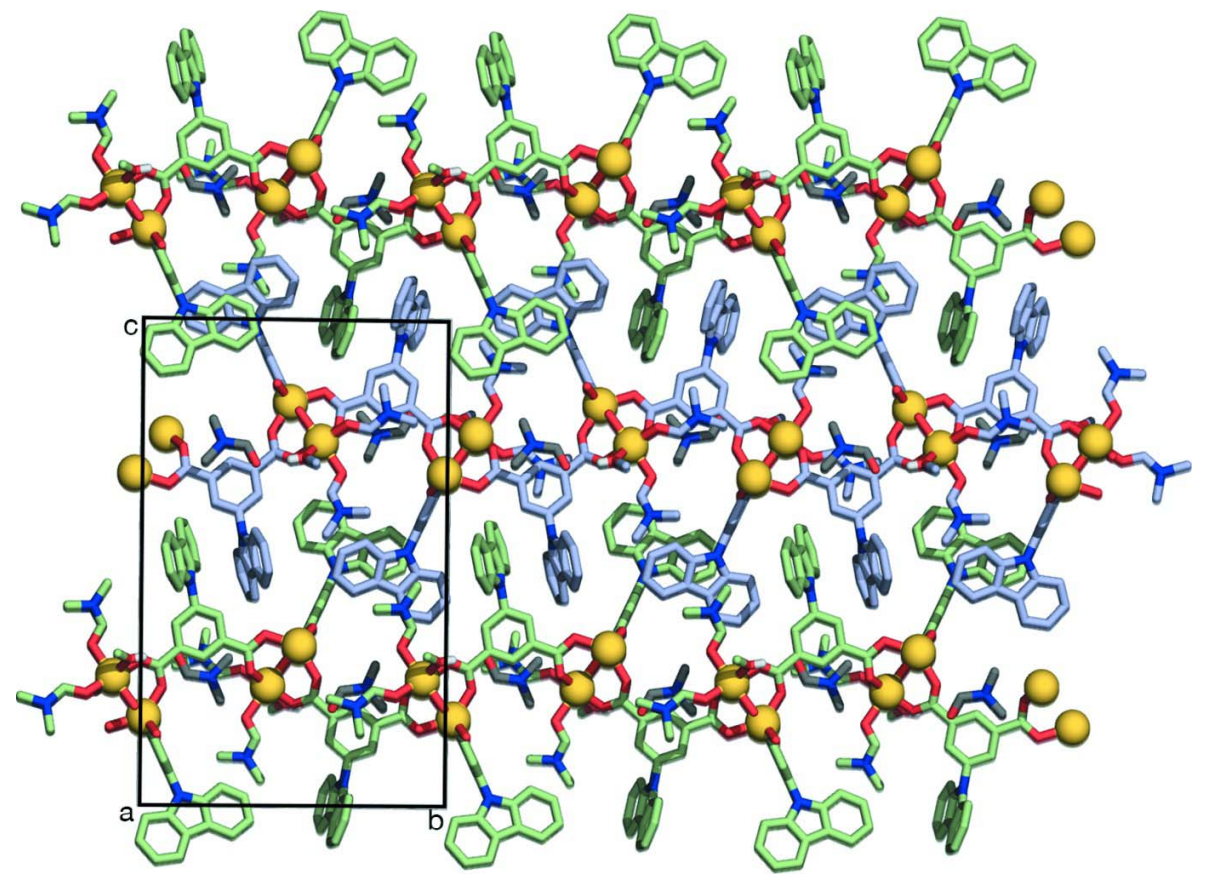

Figure 3

Crystal structure of the title complex in a projection down [100] showing the layer-like arrangement of the Zn secondary building units and the carbazole moieties parallel to (001), and the interdigitation of carbazoles to form stacked layers along [010]. Colour code as in Fig. 2. 


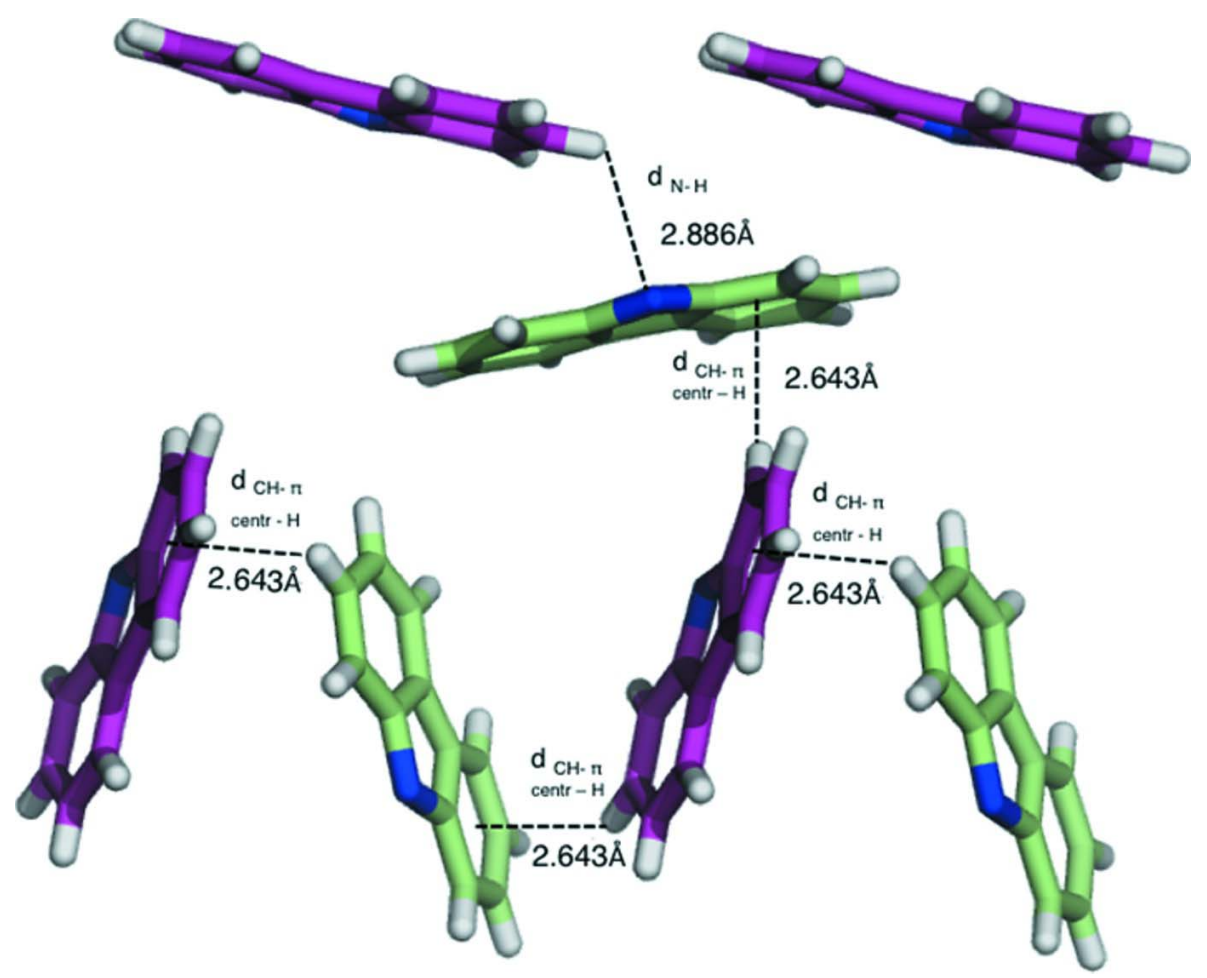

Figure 4

$\mathrm{C}-\mathrm{H}^{\cdots} \pi$ interactions between carbazole moieties in the title structure. 


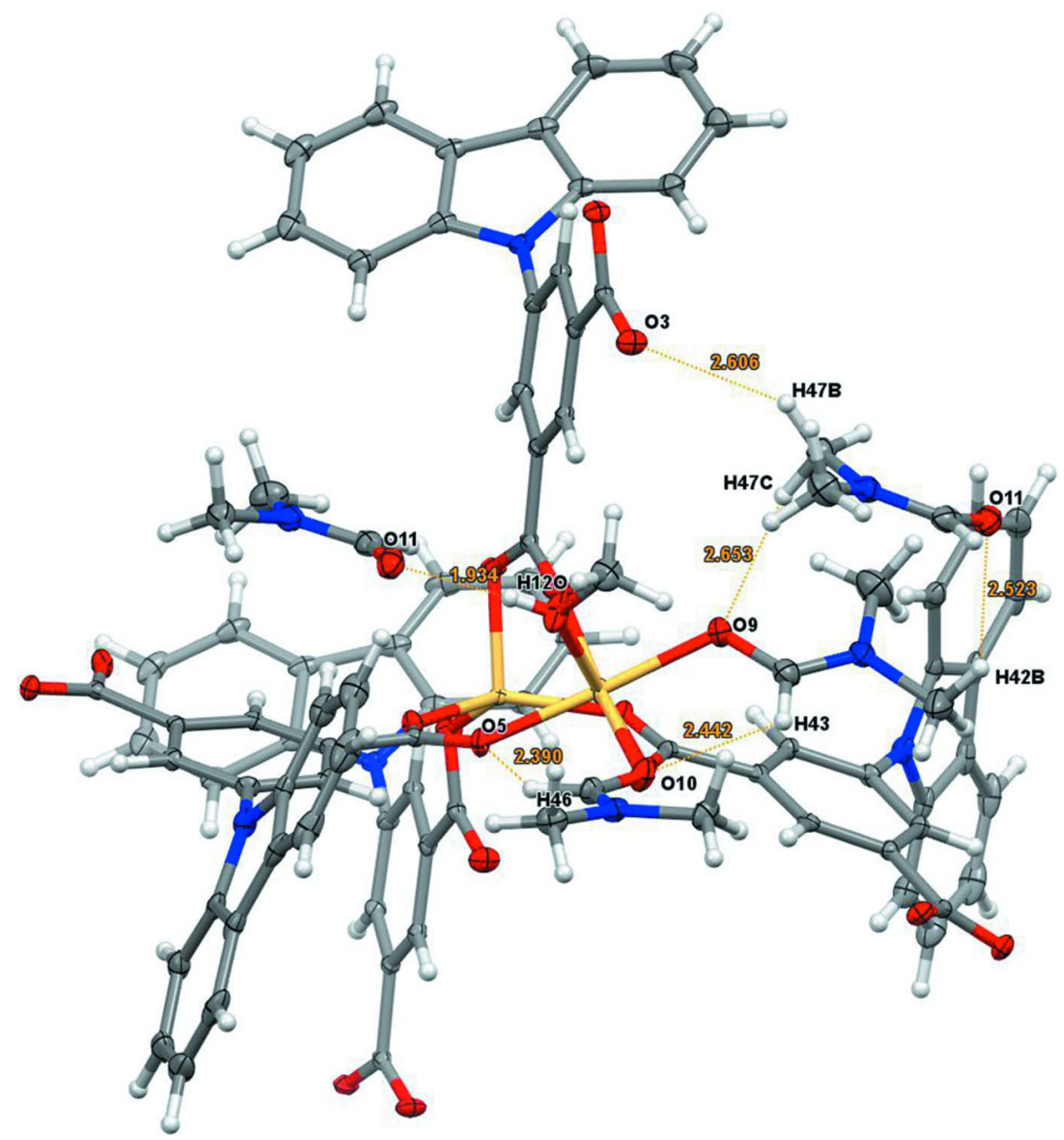

Figure 5

$\mathrm{C}-\mathrm{H} \cdots \mathrm{O}$ interactions between the coordinating DMF molecule, the DMF solvate molecule, and ligating carboxylate oxygen atoms.

Poly $\left[\left[\left[\mu_{4}-5\right.\right.\right.$ - $\left(9 H\right.$-carbazol-9-yl)isophthalato] $\left[\mu_{3}-5-(9 H\right.$-carbazol-9-yl)isophthalato $]$ bis(dimethylformamide) (methanol)dizinc] dimethylformamide monosolvate]

\section{Crystal data}

$\left[\mathrm{Zn}_{2}\left(\mathrm{C}_{20} \mathrm{H}_{11} \mathrm{NO}_{4}\right)_{2}\left(\mathrm{C}_{3} \mathrm{H}_{7} \mathrm{NO}\right)_{2}\left(\mathrm{CH}_{3} \mathrm{OH}\right)\right] \cdot \mathrm{C}_{3} \mathrm{H}_{7} \mathrm{NO}$

$M_{r}=1040.66$

Orthorhombic, $P 2{ }_{1} 2{ }_{1} 2_{1}$

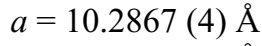

$b=17.0328(7) \AA$

$c=26.8808(11) \AA$

$V=4709.8(3) \AA^{3}$

$Z=4$

$F(000)=2152$
$D_{\mathrm{x}}=1.468 \mathrm{Mg} \mathrm{m}^{-3}$

$\mathrm{Cu} K \alpha$ radiation, $\lambda=1.54178 \AA$

Cell parameters from 9734 reflections

$\theta=5.4-68.3^{\circ}$

$\mu=1.84 \mathrm{~mm}^{-1}$

$T=100 \mathrm{~K}$

Prism, clear colourless

$0.40 \times 0.22 \times 0.22 \mathrm{~mm}$ 


\section{Data collection}

Bruker D8 Venture CMOS diffractometer

Radiation source: ImuS micro-focus source with QUAZAR optics

Mirrors monochromator

$\omega$ - and $\varphi$-scans

Absorption correction: multi-scan (SADABS; Bruker, 2015)

$T_{\min }=0.53, T_{\max }=0.68$

\section{Refinement}

Refinement on $F^{2}$

Least-squares matrix: full

$R\left[F^{2}>2 \sigma\left(F^{2}\right)\right]=0.019$

$w R\left(F^{2}\right)=0.045$

$S=1.08$

8611 reflections

629 parameters

546 restraints

0 constraints

Hydrogen site location: mixed
41928 measured reflections

8611 independent reflections

8561 reflections with $I>2 \sigma(I)$

$R_{\text {int }}=0.019$

$\theta_{\max }=68.2^{\circ}, \theta_{\min }=3.1^{\circ}$

$h=-12 \rightarrow 11$

$k=-20 \rightarrow 20$

$l=-31 \rightarrow 32$

Special details

$\mathrm{H}$-atom parameters constrained

$w=1 /\left[\sigma^{2}\left(F_{\mathrm{o}}^{2}\right)+(0.0221 P)^{2}+1.2023 P\right]$

where $P=\left(F_{\mathrm{o}}{ }^{2}+2 F_{\mathrm{c}}{ }^{2}\right) / 3$

$(\Delta / \sigma)_{\max }=0.004$

$\Delta \rho_{\max }=0.41 \mathrm{e} \AA^{-3}$

$\Delta \rho_{\min }=-0.18$ e $\AA^{-3}$

Absolute structure: Flack $x$ determined using 3716 quotients $[(\mathrm{I}+)-(\mathrm{I}-)] /[(\mathrm{I}+)+(\mathrm{I}-)]$ (Parsons et al, 2013)

Absolute structure parameter: 0.005 (3)

Geometry. All e.s.d.'s (except the e.s.d. in the dihedral angle between two 1.s. planes) are estimated using the full covariance matrix. The cell e.s.d.'s are taken into account individually in the estimation of e.s.d.'s in distances, angles and torsion angles; correlations between e.s.d.'s in cell parameters are only used when they are defined by crystal symmetry. An approximate (isotropic) treatment of cell e.s.d.'s is used for estimating e.s.d.'s involving 1.s. planes.

Fractional atomic coordinates and isotropic or equivalent isotropic displacement parameters $\left(\hat{A}^{2}\right)$

\begin{tabular}{lllll}
\hline & $x$ & $y$ & $z$ & $U_{\text {iso }} * / U_{\text {eq }}$ \\
\hline Zn1 & $0.52406(2)$ & $0.98187(2)$ & $0.67608(2)$ & $0.00831(6)$ \\
Zn2 & $0.30186(2)$ & $1.08249(2)$ & $0.75728(2)$ & $0.01117(6)$ \\
N1 & $1.01341(16)$ & $0.86300(9)$ & $0.51641(6)$ & $0.0129(3)$ \\
C1 & $0.95145(19)$ & $0.89610(12)$ & $0.47539(7)$ & $0.0146(4)$ \\
C2 & $0.9057(2)$ & $0.97254(13)$ & $0.46907(8)$ & $0.0180(4)$ \\
H2 & 0.9108 & 1.0102 & 0.4951 & $0.022^{*}$ \\
C3 & $0.8523(2)$ & $0.99110(14)$ & $0.42298(8)$ & $0.0248(5)$ \\
H3 & 0.8192 & 1.0425 & 0.4176 & $0.03 *$ \\
C4 & $0.8461(2)$ & $0.93588(15)$ & $0.38421(8)$ & $0.0277(5)$ \\
H4 & 0.8103 & 0.9506 & 0.353 & $0.033^{*}$ \\
C5 & $0.8915(2)$ & $0.86053(15)$ & $0.39113(8)$ & $0.0247(5)$ \\
H5 & 0.8872 & 0.8233 & 0.3648 & $0.03 *$ \\
C6 & $0.9441(2)$ & $0.83924(13)$ & $0.43731(8)$ & $0.0177(4)$ \\
C7 & $0.99990(19)$ & $0.76781(12)$ & $0.45673(8)$ & $0.0171(4)$ \\
C8 & $1.0156(2)$ & $0.69190(13)$ & $0.43782(8)$ & $0.0221(4)$ \\
H8 & 0.9852 & 0.679 & 0.4055 & $0.027^{*}$ \\
C9 & $1.0761(2)$ & $0.63599(13)$ & $0.46707(9)$ & $0.0238(5)$ \\
H9 & 1.0863 & 0.5841 & 0.4547 & $0.029^{*}$ \\
C10 & $1.1228(2)$ & $0.65440(13)$ & $0.51475(9)$ & $0.0223(5)$
\end{tabular}




\begin{tabular}{|c|c|c|c|c|}
\hline H10 & 1.1652 & 0.6151 & 0.5339 & $0.027^{*}$ \\
\hline $\mathrm{C} 11$ & $1.1076(2)$ & $0.72951(13)$ & $0.53442(8)$ & $0.0178(4)$ \\
\hline H11 & 1.1396 & 0.7423 & 0.5666 & $0.021 *$ \\
\hline $\mathrm{C} 12$ & $1.04404(19)$ & $0.78507(12)$ & $0.50537(7)$ & $0.0150(4)$ \\
\hline $\mathrm{C} 13$ & $1.0189(2)$ & $0.89772(10)$ & $0.56479(7)$ & $0.0119(4)$ \\
\hline $\mathrm{C} 14$ & $1.13827(19)$ & 0.91247 (11) & $0.58751(7)$ & $0.0119(4)$ \\
\hline H14 & 1.2173 & 0.8991 & 0.5713 & $0.014^{*}$ \\
\hline $\mathrm{C} 15$ & $1.13966(19)$ & $0.94734(11)$ & $0.63455(7)$ & $0.0115(4)$ \\
\hline $\mathrm{C} 16$ & $1.0238(2)$ & $0.96674(10)$ & $0.65848(7)$ & $0.0113(3)$ \\
\hline H16 & 1.0258 & 0.9911 & 0.6903 & $0.014^{*}$ \\
\hline $\mathrm{C} 17$ & $0.90537(19)$ & 0.95048 (11) & $0.63589(7)$ & $0.0107(4)$ \\
\hline $\mathrm{C} 18$ & $0.90292(19)$ & 0.91604 (11) & $0.58874(7)$ & $0.0117(4)$ \\
\hline H18 & 0.8222 & 0.9052 & 0.573 & $0.014^{*}$ \\
\hline C19 & $1.26733(18)$ & $0.96743(10)$ & $0.65857(7)$ & $0.0112(4)$ \\
\hline $\mathrm{O} 1$ & 1.36949 (13) & $0.93922(8)$ & $0.63956(5)$ & $0.0135(3)$ \\
\hline $\mathrm{O} 2$ & $1.26507(14)$ & $1.01114(9)$ & $0.69596(5)$ & $0.0166(3)$ \\
\hline $\mathrm{C} 20$ & 0.77903 (18) & 0.97111 (11) & $0.66134(7)$ & 0.0118 (4) \\
\hline $\mathrm{O} 3$ & $0.77960(14)$ & $1.01396(9)$ & $0.69850(5)$ & $0.0174(3)$ \\
\hline $\mathrm{O} 4$ & $0.67727(13)$ & $0.94091(8)$ & $0.64176(5)$ & $0.0145(3)$ \\
\hline N2 & $0.39399(18)$ & $0.83315(10)$ & $0.95005(6)$ & $0.0147(3)$ \\
\hline C21 & $0.4863(2)$ & $0.83442(11)$ & $0.98791(7)$ & $0.0155(4)$ \\
\hline $\mathrm{C} 22$ & $0.6179(2)$ & $0.81631(12)$ & $0.98552(8)$ & $0.0188(4)$ \\
\hline $\mathrm{H} 22$ & 0.6558 & 0.7959 & 0.956 & $0.023^{*}$ \\
\hline $\mathrm{C} 23$ & $0.6920(3)$ & $0.82912(12)$ & $1.02792(8)$ & $0.0245(5)$ \\
\hline $\mathrm{H} 23$ & 0.7825 & 0.8181 & 1.0272 & $0.029 *$ \\
\hline $\mathrm{C} 24$ & $0.6360(3)$ & $0.85803(13)$ & $1.07181(8)$ & $0.0270(5)$ \\
\hline $\mathrm{H} 24$ & 0.689 & 0.8669 & 1.1002 & $0.032 *$ \\
\hline $\mathrm{C} 25$ & 0.5048 (3) & $0.87372(12)$ & $1.07420(8)$ & $0.0264(5)$ \\
\hline $\mathrm{H} 25$ & 0.4673 & 0.8919 & 1.1044 & $0.032 *$ \\
\hline $\mathrm{C} 26$ & $0.4265(2)$ & $0.86281(12)$ & $1.03187(8)$ & $0.0190(4)$ \\
\hline $\mathrm{C} 27$ & 0.2928 (2) & 0.87905 (11) & $1.01955(8)$ & $0.0197(4)$ \\
\hline $\mathrm{C} 28$ & 0.1854 (3) & $0.90598(12)$ & $1.04676(8)$ & $0.0265(5)$ \\
\hline H28 & 0.1933 & 0.917 & 1.0813 & $0.032 *$ \\
\hline $\mathrm{C} 29$ & 0.0688 (3) & $0.91627(14)$ & $1.02287(10)$ & $0.0302(5)$ \\
\hline H29 & -0.0041 & 0.9346 & 1.0411 & $0.036^{*}$ \\
\hline $\mathrm{C} 30$ & 0.0554 (2) & $0.90011(13)$ & $0.97173(10)$ & $0.0281(5)$ \\
\hline H30 & -0.0264 & 0.9081 & 0.9561 & $0.034^{*}$ \\
\hline C31 & $0.1596(2)$ & $0.87272(12)$ & $0.94368(8)$ & $0.0212(4)$ \\
\hline H31 & 0.1509 & 0.8617 & 0.9092 & $0.025^{*}$ \\
\hline $\mathrm{C} 32$ & $0.2771(2)$ & $0.86227(11)$ & $0.96842(8)$ & $0.0167(4)$ \\
\hline $\mathrm{C} 33$ & $0.4234(2)$ & $0.81833(11)$ & $0.89901(7)$ & $0.0124(4)$ \\
\hline $\mathrm{C} 34$ & $0.42478(19)$ & $0.88080(11)$ & $0.86558(7)$ & $0.0121(4)$ \\
\hline H34 & 0.4032 & 0.9322 & 0.8765 & $0.015^{*}$ \\
\hline $\mathrm{C} 35$ & $0.45792(18)$ & $0.86756(11)$ & $0.81612(7)$ & $0.0109(4)$ \\
\hline $\mathrm{C} 36$ & $0.48732(19)$ & $0.79181(11)$ & $0.79992(7)$ & $0.0106(4)$ \\
\hline H36 & 0.5115 & 0.7829 & 0.7663 & $0.013 *$ \\
\hline C37 & $0.48112(19)$ & $0.72937(10)$ & $0.83313(7)$ & $0.0107(3)$ \\
\hline C38 & $0.45010(18)$ & $0.74252(11)$ & $0.88311(7)$ & $0.0118(4)$ \\
\hline
\end{tabular}




\begin{tabular}{|c|c|c|c|c|}
\hline $\mathrm{H} 38$ & 0.4474 & 0.7 & 0.9059 & $0.014 *$ \\
\hline C39 & 0.45579 (18) & $0.93481(11)$ & $0.77969(7)$ & $0.0106(4)$ \\
\hline O5 & 0.39906 (14) & $0.99586(8)$ & $0.79345(5)$ & $0.0146(3)$ \\
\hline O6 & $0.51058(13)$ & $0.92271(7)$ & $0.73846(5)$ & 0.0134 \\
\hline $\mathrm{C} 40$ & $0.50276(18)$ & $0.64683(11)$ & $0.81456(7)$ & 0.0107 (4) \\
\hline $\mathrm{O} 7$ & $0.53555(15)$ & $0.63806(8)$ & $0.77011(5)$ & 0.0153 \\
\hline O8 & $0.48208(14)$ & $0.59232(7)$ & $0.84533(5)$ & 0.0131 \\
\hline N3 & $0.18493(18)$ & $1.29637(10)$ & $0.69906(7)$ & 0.0203 \\
\hline C41 & $0.1585(3)$ & $1.28253(18)$ & $0.64698(9)$ & $0.0359(6)$ \\
\hline H41A & 0.1585 & 1.2259 & 0.6405 & $0.043^{*}$ \\
\hline H41B & 0.2258 & 1.3078 & 0.6267 & $0.043 *$ \\
\hline $\mathrm{H} 41 \mathrm{C}$ & 0.0733 & 1.3045 & 0.6384 & $0.043^{*}$ \\
\hline $\mathrm{C} 42$ & 0.1993 & $1.37942(14)$ & $0.71429(12)$ & $0.0368(6)$ \\
\hline $\mathrm{H} 42 \mathrm{~A}$ & 0.2282 & 1.3818 & 0.749 & $0.044^{*}$ \\
\hline H42B & 0.1156 & 1.4063 & 0.711 & $0.044 *$ \\
\hline $\mathrm{H} 42 \mathrm{C}$ & 0.2638 & 1.4051 & 0.693 & $0.044^{*}$ \\
\hline $\mathrm{C} 43$ & 0.2047 (2) & $1.23963(13)$ & $0.73133(9)$ & $0.0231(4)$ \\
\hline $\mathrm{H} 43$ & 0.2304 & 1.2537 & 0.7641 & $0.028 *$ \\
\hline O9 & $0.19190(17)$ & $1.16868(9)$ & $0.72161(6)$ & $0.0249(3)$ \\
\hline N4 & $0.23401(18)$ & $1.14908(10)$ & $0.90142(6)$ & $0.0165(4)$ \\
\hline C44 & $0.1897(2)$ & $1.23038(12)$ & $0.90000(8)$ & $0.0212(4)$ \\
\hline $\mathrm{H} 44 \mathrm{~A}$ & 0.2421 & 1.2599 & 0.876 & $0.025^{*}$ \\
\hline H44B & 0.1991 & 1.2539 & 0.9331 & $0.025^{*}$ \\
\hline $\mathrm{H} 44 \mathrm{C}$ & 0.0982 & 1.2319 & 0.89 & $0.025^{*}$ \\
\hline $\mathrm{C} 45$ & $0.2081(2)$ & $1.10314(13)$ & $0.94622(7)$ & $0.0206(4)$ \\
\hline $\mathrm{H} 45 \mathrm{~A}$ & 0.1143 & 1.0943 & 0.9494 & $0.025^{*}$ \\
\hline H45B & 0.2396 & 1.1318 & 0.9754 & $0.025^{*}$ \\
\hline $\mathrm{H} 45 \mathrm{C}$ & 0.2529 & 1.0525 & 0.9439 & $0.025^{*}$ \\
\hline $\mathrm{C} 46$ & $0.2883(2)$ & 1.11725 (12) & $0.86199(7)$ & $0.0170(4)$ \\
\hline $\mathrm{H} 46$ & 0.317 & 1.0644 & 0.8644 & $0.02 *$ \\
\hline $\mathrm{O} 10$ & $0.30448(16)$ & $1.15212(8)$ & $0.82162(5)$ & 0.0193 \\
\hline N5 & $0.18348(18)$ & $0.76557(10)$ & $0.75996(7)$ & 0.0198 \\
\hline $\mathrm{C} 47$ & $0.1482(2)$ & $0.70971(13)$ & $0.79858(9)$ & $0.0228(5)$ \\
\hline H47A & 0.1474 & 0.7363 & 0.8309 & $0.027^{*}$ \\
\hline H47B & 0.2117 & 0.6668 & 0.7992 & $0.027^{*}$ \\
\hline $\mathrm{H} 47 \mathrm{C}$ & 0.0616 & 0.6884 & 0.7916 & $0.027^{*}$ \\
\hline $\mathrm{C} 48$ & $0.2438(3)$ & $0.73480(16)$ & $0.71458(9)$ & $0.0299(5)$ \\
\hline H48A & 0.2654 & 0.7784 & 0.6923 & $0.036^{*}$ \\
\hline H48B & 0.183 & 0.6992 & 0.6978 & $0.036^{*}$ \\
\hline $\mathrm{H} 48 \mathrm{C}$ & 0.3232 & 0.7062 & 0.7232 & $0.036^{*}$ \\
\hline C49 & $0.1679(2)$ & $0.84238(13)$ & $0.76636(8)$ & 0.0218 \\
\hline H49 & 0.1952 & 0.876 & 0.7402 & $0.026^{*}$ \\
\hline O11 & $0.12041(16)$ & $0.87346(9)$ & $0.80389(6)$ & 0.0238 \\
\hline $\mathrm{C} 50$ & $0.0105(2)$ & 1.06679 (13) & $0.80194(9)$ & $0.0265(5)$ \\
\hline H50A & 0.0221 & 1.1239 & 0.8027 & $0.032 *$ \\
\hline H50B & -0.0201 & 1.0487 & 0.8345 & $0.032 *$ \\
\hline H50C & -0.0536 & 1.053 & 0.7764 & $0.032 *$ \\
\hline $\mathrm{O} 12$ & $0.13112(15)$ & $1.03020(9)$ & $0.79064(6)$ & 0.0238 \\
\hline
\end{tabular}




$\begin{array}{lllll}\mathrm{H} 12 \mathrm{O} & 0.1229 & 0.9862 & 0.7954 & 0.029 *\end{array}$

Atomic displacement parameters $\left(\AA^{2}\right)$

\begin{tabular}{|c|c|c|c|c|c|c|}
\hline & $U^{11}$ & $U^{22}$ & $U^{33}$ & $U^{12}$ & $U^{13}$ & $U^{23}$ \\
\hline $\mathrm{Zn} 1$ & $0.00790(11)$ & $0.00827(11)$ & 0.00877 (11) & $0.00017(9)$ & $0.00090(9)$ & $0.00012(9)$ \\
\hline $\mathrm{Zn} 2$ & $0.01339(12)$ & $0.00866(11)$ & $0.01146(12)$ & $0.00126(9)$ & $0.00188(9)$ & $-0.00029(9)$ \\
\hline N1 & $0.0120(8)$ & $0.0150(7)$ & $0.0118(7)$ & $0.0003(6)$ & $0.0004(6)$ & -0.0050 \\
\hline $\mathrm{C} 1$ & $0.0096(9)$ & $0.0205(10)$ & $0.0136(9)$ & $-0.0038(7)$ & $0.0005(7)$ & $-0.0014(7)$ \\
\hline $\mathrm{C} 2$ & $0.0167(10)$ & $0.0191(10)$ & $0.0184(10)$ & $-0.0045(8)$ & $-0.0014(8)$ & $-0.0004(8)$ \\
\hline $\mathrm{C} 3$ & $0.0262(12)$ & $0.0244(11)$ & $0.0238(11)$ & $-0.0048(9)$ & $-0.0047(9)$ & $0.0069(9)$ \\
\hline $\mathrm{C} 4$ & $0.0296(13)$ & $0.0364(13)$ & $0.0172(11)$ & $-0.0084(10)$ & $-0.0071(9)$ & $0.0048(9)$ \\
\hline $\mathrm{C} 5$ & $0.0254(12)$ & $0.0343(12)$ & $0.0143(10)$ & $-0.0077(10)$ & $-0.0024(9)$ & $-0.0064(9)$ \\
\hline C6 & $0.0140(10)$ & $0.0240(10)$ & $0.0149(9)$ & $-0.0051(8)$ & $0.0017(7)$ & $-0.0052(8)$ \\
\hline $\mathrm{C} 7$ & $0.0112(10)$ & $0.0222(10)$ & $0.0178(9)$ & $-0.0035(8)$ & $0.0034(7)$ & $-0.0074(8)$ \\
\hline $\mathrm{C} 8$ & $0.0193(11)$ & $0.0234(10)$ & $0.0236(10)$ & -0.0057 (9) & $0.0057(9)$ & $-0.0122(8)$ \\
\hline $\mathrm{C} 9$ & 0.0197 (11) & $0.0186(10)$ & $0.0331(12)$ & $-0.0035(9)$ & $0.0119(9)$ & $-0.0110(9)$ \\
\hline $\mathrm{C} 10$ & $0.0139(10)$ & 0.0188 (10) & $0.0342(12)$ & $0.0015(8)$ & $0.0079(9)$ & -0.0025 (9) \\
\hline C11 & $0.0120(9)$ & $0.0200(10)$ & $0.0214(10)$ & $0.0001(8)$ & $0.0025(8)$ & $-0.0047(8)$ \\
\hline $\mathrm{C} 12$ & $0.0103(9)$ & $0.0174(9)$ & $0.0174(9)$ & $-0.0020(7)$ & $0.0042(7)$ & $-0.0055(7)$ \\
\hline C13 & $0.0128(9)$ & $0.0111(8)$ & $0.0119(8)$ & $-0.0007(7)$ & $-0.0006(7)$ & $-0.0014(7)$ \\
\hline $\mathrm{C} 14$ & $0.0100(9)$ & $0.0117(9)$ & $0.0140(9)$ & $0.0002(7)$ & $0.0009(7)$ & $-0.0005(7)$ \\
\hline $\mathrm{C} 15$ & $0.0113(9)$ & $0.0091(8)$ & $0.0141(9)$ & $-0.0005(7)$ & $0.0000(7)$ & $0.0017(7)$ \\
\hline $\mathrm{C} 16$ & $0.0138(9)$ & $0.0095(8)$ & $0.0106(8)$ & $-0.0002(7)$ & $0.0010(7)$ & $0.0008(6)$ \\
\hline $\mathrm{C} 17$ & $0.0112(9)$ & $0.0090(8)$ & $0.0118(9)$ & $-0.0002(7)$ & $0.0016(7)$ & $0.0017(7)$ \\
\hline C18 & $0.0092(9)$ & $0.0107(8)$ & $0.0152(9)$ & $-0.0007(7)$ & $-0.0014(7)$ & $0.0009(7)$ \\
\hline C19 & $0.0106(9)$ & $0.0104(9)$ & $0.0127(9)$ & $-0.0005(7)$ & $0.0001(7)$ & $0.0032(7)$ \\
\hline $\mathrm{O} 1$ & $0.0084(7)$ & $0.0156(7)$ & $0.0166(7)$ & $0.0000(5)$ & $-0.0013(5)$ & $-0.0023(5)$ \\
\hline $\mathrm{O} 2$ & $0.0141(7)$ & $0.0206(7)$ & $0.0151(7)$ & $-0.0022(6)$ & $-0.0027(5)$ & $-0.0063(6)$ \\
\hline $\mathrm{C} 20$ & $0.0109(9)$ & $0.0104(8)$ & $0.0140(9)$ & $0.0015(7)$ & $0.0021(7)$ & $0.0032(7)$ \\
\hline $\mathrm{O} 3$ & $0.0146(7)$ & $0.0222(7)$ & $0.0155(7)$ & $0.0014(6)$ & $0.0021(5)$ & $-0.0068(6)$ \\
\hline $\mathrm{O} 4$ & $0.0099(7)$ & $0.0173(7)$ & $0.0163(7)$ & $0.0003(5)$ & $0.0016(5)$ & $-0.0037(5)$ \\
\hline N2 & $0.0219(9)$ & $0.0129(8)$ & $0.0092(8)$ & $0.0017(7)$ & $0.0028(7)$ & $-0.0004(6)$ \\
\hline $\mathrm{C} 21$ & $0.0284(11)$ & $0.0080(8)$ & $0.0100(8)$ & $-0.0010(8)$ & $-0.0010(8)$ & $0.0009(7)$ \\
\hline $\mathrm{C} 22$ & 0.0277 (12) & $0.0129(9)$ & $0.0157(10)$ & $0.0024(8)$ & $-0.0009(8)$ & $0.0016(8)$ \\
\hline $\mathrm{C} 23$ & $0.0364(13)$ & $0.0148(9)$ & $0.0221(11)$ & $0.0026(10)$ & $-0.0090(10)$ & $0.0031(8)$ \\
\hline $\mathrm{C} 24$ & $0.0468(15)$ & $0.0184(10)$ & $0.0159(10)$ & $-0.0035(10)$ & $-0.0105(10)$ & $0.0022(8)$ \\
\hline $\mathrm{C} 25$ & 0.0545 (17) & $0.0136(9)$ & $0.0110(9)$ & $-0.0080(10)$ & $0.0022(10)$ & $-0.0012(7)$ \\
\hline $\mathrm{C} 26$ & $0.0357(12)$ & $0.0091(9)$ & $0.0122(9)$ & $-0.0044(8)$ & $0.0049(8)$ & $0.0001(7)$ \\
\hline $\mathrm{C} 27$ & $0.0348(12)$ & $0.0099(9)$ & $0.0145(9)$ & $-0.0059(9)$ & $0.0092(9)$ & $-0.0029(7)$ \\
\hline $\mathrm{C} 28$ & $0.0403(14)$ & $0.0167(10)$ & $0.0226(11)$ & $-0.0058(10)$ & $0.0180(10)$ & $-0.0065(8)$ \\
\hline $\mathrm{C} 29$ & $0.0340(13)$ & $0.0191(10)$ & $0.0376(13)$ & $-0.0027(10)$ & $0.0224(11)$ & $-0.0056(10)$ \\
\hline $\mathrm{C} 30$ & $0.0239(12)$ & $0.0215(11)$ & $0.0388(13)$ & 0.0007 (9) & $0.0104(10)$ & $-0.0011(10)$ \\
\hline C31 & $0.0243(11)$ & $0.0159(10)$ & $0.0233(11)$ & $-0.0007(8)$ & $0.0063(9)$ & $-0.0007(8)$ \\
\hline $\mathrm{C} 32$ & $0.0251(11)$ & $0.0085(8)$ & $0.0165(10)$ & $-0.0006(8)$ & $0.0090(8)$ & -0.0009 (7) \\
\hline $\mathrm{C} 33$ & $0.0147(9)$ & $0.0145(9)$ & $0.0079(8)$ & $0.0004(8)$ & $0.0004(7)$ & $-0.0019(7)$ \\
\hline C34 & $0.0130(9)$ & $0.0104(9)$ & $0.0131(9)$ & $0.0005(7)$ & $0.0009(7)$ & $-0.0013(7)$ \\
\hline $\mathrm{C} 35$ & $0.0099(9)$ & $0.0112(8)$ & $0.0117(9)$ & $0.0000(7)$ & $-0.0011(7)$ & $0.0009(7)$ \\
\hline
\end{tabular}




$\begin{array}{lllllll}\text { C36 } & 0.0095(9) & 0.0133(8) & 0.0091(8) & 0.0007(7) & -0.0007(7) & -0.0009(7) \\ \text { C37 } & 0.0088(8) & 0.0113(8) & 0.0119(8) & 0.0009(7) & -0.0009(7) & -0.0007(7) \\ \text { C38 } & 0.0117(9) & 0.0117(9) & 0.0119(9) & -0.0007(7) & 0.0007(7) & 0.0022(7) \\ \text { C39 } & 0.0098(9) & 0.0110(9) & 0.0110(8) & -0.0005(7) & -0.0022(7) & 0.0007(7) \\ \text { O5 } & 0.0215(7) & 0.0096(6) & 0.0127(6) & 0.0037(5) & 0.0014(5) & 0.0006(5) \\ \text { O6 } & 0.0155(7) & 0.0140(6) & 0.0106(6) & 0.0024(5) & 0.0033(5) & 0.0030(5) \\ \text { C40 } & 0.0085(9) & 0.0117(8) & 0.0120(9) & 0.0009(7) & -0.0018(7) & -0.0006(7) \\ \text { O7 } & 0.0211(7) & 0.0141(6) & 0.0107(6) & 0.0067(6) & 0.0010(6) & -0.0020(5) \\ \text { O8 } & 0.0160(7) & 0.0097(6) & 0.0136(6) & 0.0005(5) & 0.0011(5) & -0.0010(5) \\ \text { N3 } & 0.0154(9) & 0.0186(8) & 0.0270(9) & 0.0020(7) & -0.0017(7) & 0.0010(7) \\ \text { C41 } & 0.0376(15) & 0.0446(15) & 0.0256(12) & 0.0053(12) & -0.0028(10) & 0.0056(11) \\ \text { C42 } & 0.0266(13) & 0.0203(11) & 0.0634(18) & 0.0022(10) & -0.0086(13) & -0.0019(11) \\ \text { C43 } & 0.0193(10) & 0.0224(10) & 0.0276(11) & 0.0038(9) & -0.0044(9) & -0.0020(9) \\ \text { O9 } & 0.0241(8) & 0.0159(7) & 0.0349(8) & 0.0039(6) & -0.0082(7) & 0.0007(6) \\ \text { N4 } & 0.0170(9) & 0.0172(8) & 0.0152(8) & -0.0004(7) & 0.0013(7) & -0.0041(7) \\ \text { C44 } & 0.0235(11) & 0.0173(10) & 0.0229(10) & 0.0020(9) & 0.0041(9) & -0.0065(8) \\ \text { C45 } & 0.0227(11) & 0.0245(10) & 0.0146(10) & -0.0006(9) & 0.0028(8) & -0.0007(8) \\ \text { C46 } & 0.0201(10) & 0.0151(9) & 0.0156(10) & 0.0021(8) & 0.0006(8) & -0.0024(7) \\ \text { O10 } & 0.0295(8) & 0.0143(6) & 0.0142(6) & 0.0018(6) & 0.0048(7) & -0.0035(5) \\ \text { N5 } & 0.0182(9) & 0.0209(8) & 0.0202(8) & -0.0020(7) & -0.0010(7) & 0.0009(7) \\ \text { C47 } & 0.0216(11) & 0.0207(10) & 0.0260(11) & -0.0009(9) & -0.0006(9) & 0.0025(9) \\ \text { C48 } & 0.0301(13) & 0.0351(13) & 0.0247(12) & -0.0039(11) & 0.0027(10) & -0.0041(10) \\ \text { C49 } & 0.0163(10) & 0.0234(10) & 0.0257(11) & -0.0037(8) & -0.0019(8) & 0.0057(9) \\ \text { O11 } & 0.0189(8) & 0.0186(7) & 0.0339(9) & -0.0006(6) & 0.0013(7) & 0.0009(6) \\ \text { C50 } & 0.0168(11) & 0.0255(11) & 0.0374(12) & 0.0029(9) & 0.0072(9) & -0.0048(9) \\ \text { O12 } & 0.0192(8) & 0.0142(7) & 0.0381(9) & 0.0015(6) & 0.0150(7) & 0.0019(6)\end{array}$

Geometric parameters $\left(\AA,{ }^{\circ}\right)$

\begin{tabular}{llll}
\hline $\mathrm{Zn} 1-\mathrm{O} 4$ & $1.9548(14)$ & $\mathrm{C} 27-\mathrm{C} 28$ & $1.402(3)$ \\
$\mathrm{Zn} 1-\mathrm{O} 6$ & $1.9614(13)$ & $\mathrm{C} 27-\mathrm{C} 32$ & $1.413(3)$ \\
$\mathrm{Zn} 1-\mathrm{O} 8^{\mathrm{i}}$ & $1.9683(13)$ & $\mathrm{C} 28-\mathrm{C} 29$ & $1.372(4)$ \\
$\mathrm{Zn} 1-\mathrm{O} 1^{\mathrm{ii}}$ & $2.0047(14)$ & $\mathrm{C} 28-\mathrm{H} 28$ & 0.95 \\
$\mathrm{Zn} 2-\mathrm{O} 5$ & $2.0303(14)$ & $\mathrm{C} 29-\mathrm{C} 30$ & $1.409(4)$ \\
$\mathrm{Zn} 2-\mathrm{O} 7^{\mathrm{i}}$ & $2.0580(14)$ & $\mathrm{C} 29-\mathrm{H} 29$ & 0.95 \\
$\mathrm{Zn} 2-\mathrm{O} 2^{\mathrm{ii}}$ & $2.0826(14)$ & $\mathrm{C} 30-\mathrm{C} 31$ & $1.391(3)$ \\
$\mathrm{Zn} 2-\mathrm{O} 9$ & $2.0867(15)$ & $\mathrm{C} 30-\mathrm{H} 30$ & 0.95 \\
$\mathrm{Zn} 2-\mathrm{O} 10$ & $2.0974(14)$ & $\mathrm{C} 31-\mathrm{C} 32$ & $1.391(3)$ \\
$\mathrm{Zn} 2-\mathrm{O} 12$ & $2.1638(15)$ & $\mathrm{C} 31-\mathrm{H} 31$ & 0.95 \\
$\mathrm{~N} 1-\mathrm{C} 1$ & $1.393(3)$ & $\mathrm{C} 33-\mathrm{C} 38$ & $1.388(3)$ \\
$\mathrm{N} 1-\mathrm{C} 12$ & $1.396(3)$ & $\mathrm{C} 33-\mathrm{C} 34$ & $1.393(3)$ \\
$\mathrm{N} 1-\mathrm{C} 13$ & $1.430(2)$ & $\mathrm{C} 34-\mathrm{C} 35$ & $1.391(3)$ \\
$\mathrm{C} 1-\mathrm{C} 2$ & $1.395(3)$ & $\mathrm{C} 34-\mathrm{H} 34$ & 0.95 \\
$\mathrm{C} 1-\mathrm{C} 6$ & $1.411(3)$ & $\mathrm{C} 35-\mathrm{C} 36$ & $1.395(3)$ \\
$\mathrm{C} 2-\mathrm{C} 3$ & $1.392(3)$ & $\mathrm{C} 35-\mathrm{C} 39$ & $1.507(3)$ \\
$\mathrm{C} 2-\mathrm{H} 2$ & 0.95 & $\mathrm{C} 36-\mathrm{C} 37$ & $1.390(3)$ \\
$\mathrm{C} 3-\mathrm{C} 4$ & $1.405(3)$ & $\mathrm{C} 36-\mathrm{H} 36$ & 0.95 \\
$\mathrm{C} 3-\mathrm{H} 3$ & 0.95 & $\mathrm{C} 37-\mathrm{C} 38$ & $1.399(3)$
\end{tabular}




\begin{tabular}{|c|c|c|c|}
\hline $\mathrm{C} 4-\mathrm{C} 5$ & $1.378(4)$ & $\mathrm{C} 37-\mathrm{C} 40$ & $1.508(2)$ \\
\hline $\mathrm{C} 4-\mathrm{H} 4$ & 0.95 & $\mathrm{C} 38-\mathrm{H} 38$ & 0.95 \\
\hline $\mathrm{C} 5-\mathrm{C} 6$ & $1.402(3)$ & $\mathrm{C} 39-\mathrm{O} 5$ & $1.248(2)$ \\
\hline C5-H5 & 0.95 & $\mathrm{C} 39-\mathrm{O} 6$ & $1.260(2)$ \\
\hline $\mathrm{C} 6-\mathrm{C} 7$ & $1.443(3)$ & $\mathrm{C} 40-\mathrm{O} 7$ & $1.250(2)$ \\
\hline $\mathrm{C} 7-\mathrm{C} 8$ & $1.399(3)$ & $\mathrm{C} 40-\mathrm{O} 8$ & $1.262(2)$ \\
\hline $\mathrm{C} 7-\mathrm{C} 12$ & $1.415(3)$ & $\mathrm{O} 7-\mathrm{Zn} 2^{\mathrm{iv}}$ & $2.0580(14)$ \\
\hline $\mathrm{C} 8-\mathrm{C} 9$ & $1.383(4)$ & $\mathrm{O} 8-\mathrm{Zn} 1^{\mathrm{iv}}$ & $1.9683(13)$ \\
\hline $\mathrm{C} 8-\mathrm{H} 8$ & 0.95 & $\mathrm{~N} 3-\mathrm{C} 43$ & $1.315(3)$ \\
\hline $\mathrm{C} 9-\mathrm{C} 10$ & $1.404(3)$ & $\mathrm{N} 3-\mathrm{C} 41$ & $1.445(3)$ \\
\hline С9-H9 & 0.95 & $\mathrm{~N} 3-\mathrm{C} 42$ & $1.480(3)$ \\
\hline $\mathrm{C} 10-\mathrm{C} 11$ & $1.393(3)$ & $\mathrm{C} 41-\mathrm{H} 41 \mathrm{~A}$ & 0.98 \\
\hline $\mathrm{C} 10-\mathrm{H} 10$ & 0.95 & $\mathrm{C} 41-\mathrm{H} 41 \mathrm{~B}$ & 0.98 \\
\hline $\mathrm{C} 11-\mathrm{C} 12$ & $1.390(3)$ & $\mathrm{C} 41-\mathrm{H} 41 \mathrm{C}$ & 0.98 \\
\hline $\mathrm{C} 11-\mathrm{H} 11$ & 0.95 & $\mathrm{C} 42-\mathrm{H} 42 \mathrm{~A}$ & 0.98 \\
\hline $\mathrm{C} 13-\mathrm{C} 18$ & $1.391(3)$ & $\mathrm{C} 42-\mathrm{H} 42 \mathrm{~B}$ & 0.98 \\
\hline $\mathrm{C} 13-\mathrm{C} 14$ & $1.395(3)$ & $\mathrm{C} 42-\mathrm{H} 42 \mathrm{C}$ & 0.98 \\
\hline $\mathrm{C} 14-\mathrm{C} 15$ & $1.397(3)$ & $\mathrm{C} 43-\mathrm{O} 9$ & $1.243(3)$ \\
\hline C14-H14 & 0.95 & $\mathrm{C} 43-\mathrm{H} 43$ & 0.95 \\
\hline $\mathrm{C} 15-\mathrm{C} 16$ & $1.395(3)$ & $\mathrm{N} 4-\mathrm{C} 46$ & $1.315(3)$ \\
\hline $\mathrm{C} 15-\mathrm{C} 19$ & $1.503(3)$ & $\mathrm{N} 4-\mathrm{C} 44$ & $1.458(3)$ \\
\hline $\mathrm{C} 16-\mathrm{C} 17$ & $1.389(3)$ & $\mathrm{N} 4-\mathrm{C} 45$ & $1.461(3)$ \\
\hline C16-H16 & 0.95 & $\mathrm{C} 44-\mathrm{H} 44 \mathrm{~A}$ & 0.98 \\
\hline $\mathrm{C} 17-\mathrm{C} 18$ & $1.397(3)$ & C44-H44B & 0.98 \\
\hline $\mathrm{C} 17-\mathrm{C} 20$ & $1.510(3)$ & $\mathrm{C} 44-\mathrm{H} 44 \mathrm{C}$ & 0.98 \\
\hline C18-H18 & 0.95 & $\mathrm{C} 45-\mathrm{H} 45 \mathrm{~A}$ & 0.98 \\
\hline $\mathrm{C} 19-\mathrm{O} 2$ & $1.251(2)$ & $\mathrm{C} 45-\mathrm{H} 45 \mathrm{~B}$ & 0.98 \\
\hline $\mathrm{C} 19-\mathrm{O} 1$ & $1.263(2)$ & $\mathrm{C} 45-\mathrm{H} 45 \mathrm{C}$ & 0.98 \\
\hline $\mathrm{O} 1-\mathrm{Zn} 1^{\mathrm{iii}}$ & 2.0047 (14) & $\mathrm{C} 46-\mathrm{O} 10$ & $1.248(3)$ \\
\hline $\mathrm{O} 2-\mathrm{Zn} 2^{\mathrm{iii}}$ & $2.0825(14)$ & $\mathrm{C} 46-\mathrm{H} 46$ & 0.95 \\
\hline $\mathrm{C} 20-\mathrm{O} 3$ & $1.237(2)$ & $\mathrm{N} 5-\mathrm{C} 49$ & $1.329(3)$ \\
\hline $\mathrm{C} 20-\mathrm{O} 4$ & $1.279(2)$ & $\mathrm{N} 5-\mathrm{C} 47$ & $1.454(3)$ \\
\hline $\mathrm{N} 2-\mathrm{C} 32$ & $1.391(3)$ & N5-C48 & $1.465(3)$ \\
\hline $\mathrm{N} 2-\mathrm{C} 21$ & $1.392(3)$ & $\mathrm{C} 47-\mathrm{H} 47 \mathrm{~A}$ & 0.98 \\
\hline $\mathrm{N} 2-\mathrm{C} 33$ & $1.427(2)$ & $\mathrm{C} 47-\mathrm{H} 47 \mathrm{~B}$ & 0.98 \\
\hline $\mathrm{C} 21-\mathrm{C} 22$ & $1.390(3)$ & C47-H47C & 0.98 \\
\hline $\mathrm{C} 21-\mathrm{C} 26$ & $1.417(3)$ & $\mathrm{C} 48-\mathrm{H} 48 \mathrm{~A}$ & 0.98 \\
\hline $\mathrm{C} 22-\mathrm{C} 23$ & $1.388(3)$ & $\mathrm{C} 48-\mathrm{H} 48 \mathrm{~B}$ & 0.98 \\
\hline $\mathrm{C} 22-\mathrm{H} 22$ & 0.95 & $\mathrm{C} 48-\mathrm{H} 48 \mathrm{C}$ & 0.98 \\
\hline $\mathrm{C} 23-\mathrm{C} 24$ & $1.402(4)$ & $\mathrm{C} 49-\mathrm{O} 11$ & $1.240(3)$ \\
\hline $\mathrm{C} 23-\mathrm{H} 23$ & 0.95 & C49-H49 & 0.95 \\
\hline $\mathrm{C} 24-\mathrm{C} 25$ & $1.377(4)$ & $\mathrm{C} 50-\mathrm{O} 12$ & $1.421(3)$ \\
\hline $\mathrm{C} 24-\mathrm{H} 24$ & 0.95 & $\mathrm{C} 50-\mathrm{H} 50 \mathrm{~A}$ & 0.98 \\
\hline $\mathrm{C} 25-\mathrm{C} 26$ & $1.406(3)$ & $\mathrm{C} 50-\mathrm{H} 50 \mathrm{~B}$ & 0.98 \\
\hline $\mathrm{C} 25-\mathrm{H} 25$ & 0.95 & $\mathrm{C} 50-\mathrm{H} 50 \mathrm{C}$ & 0.98 \\
\hline $\mathrm{C} 26-\mathrm{C} 27$ & $1.442(4)$ & $\mathrm{O} 12-\mathrm{H} 12 \mathrm{O}$ & 0.7642 \\
\hline $\mathrm{O} 4-\mathrm{Zn} 1-\mathrm{O} 6$ & $106.09(6)$ & $\mathrm{C} 32-\mathrm{C} 27-\mathrm{C} 26$ & $107.09(19)$ \\
\hline
\end{tabular}




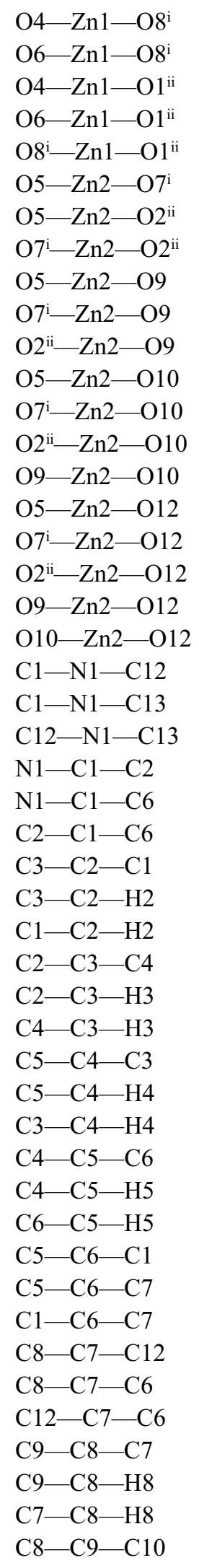

$103.24(6)$

$137.62(5)$

$106.21(5)$

$100.16(6)$

$100.24(6)$

96.05 (6)

$92.55(6)$

97.64 (5)

$176.68(7)$

$87.28(6)$

$87.04(6)$

$90.56(6)$

$91.40(6)$

170.07 (6)

$89.32(6)$

$84.38(6)$

$175.73(6)$

$86.58(6)$

$92.30(7)$

84.34 (6)

$108.65(16)$

$124.80(16)$

$125.29(16)$

$128.95(18)$

$108.73(18)$

$122.29(19)$

117.0 (2)

121.5

121.5

121.7 (2)

119.1

119.1

120.5 (2)

119.7

119.7

119.4 (2)

120.3

120.3

$119.1(2)$

$133.8(2)$

107.15 (18)

119.4 (2)

$133.9(2)$

106.67 (17)

118.8 (2)

120.6

120.6

121.3 (2)
$\mathrm{C} 29-\mathrm{C} 28-\mathrm{C} 27$

$\mathrm{C} 29-\mathrm{C} 28-\mathrm{H} 28$

$\mathrm{C} 27-\mathrm{C} 28-\mathrm{H} 28$

$\mathrm{C} 28-\mathrm{C} 29-\mathrm{C} 30$

$\mathrm{C} 28-\mathrm{C} 29-\mathrm{H} 29$

$\mathrm{C} 30-\mathrm{C} 29-\mathrm{H} 29$

$\mathrm{C} 31-\mathrm{C} 30-\mathrm{C} 29$

$\mathrm{C} 31-\mathrm{C} 30-\mathrm{H} 30$

$\mathrm{C} 29-\mathrm{C} 30-\mathrm{H} 30$

$\mathrm{C} 30-\mathrm{C} 31-\mathrm{C} 32$

$\mathrm{C} 30-\mathrm{C} 31-\mathrm{H} 31$

$\mathrm{C} 32-\mathrm{C} 31-\mathrm{H} 31$

$\mathrm{C} 31-\mathrm{C} 32-\mathrm{N} 2$

$\mathrm{C} 31-\mathrm{C} 32-\mathrm{C} 27$

$\mathrm{N} 2-\mathrm{C} 32-\mathrm{C} 27$

$\mathrm{C} 38-\mathrm{C} 33-\mathrm{C} 34$

$\mathrm{C} 38-\mathrm{C} 33-\mathrm{N} 2$

$\mathrm{C} 34-\mathrm{C} 33-\mathrm{N} 2$

$\mathrm{C} 35-\mathrm{C} 34-\mathrm{C} 33$

$\mathrm{C} 35-\mathrm{C} 34-\mathrm{H} 34$

C $33-\mathrm{C} 34-\mathrm{H} 34$

$\mathrm{C} 34-\mathrm{C} 35-\mathrm{C} 36$

C34-C35-C39

$\mathrm{C} 36-\mathrm{C} 35-\mathrm{C} 39$

$\mathrm{C} 37-\mathrm{C} 36-\mathrm{C} 35$

C37- C36-H36

$\mathrm{C} 35-\mathrm{C} 36-\mathrm{H} 36$

$\mathrm{C} 36-\mathrm{C} 37-\mathrm{C} 38$

$\mathrm{C} 36-\mathrm{C} 37-\mathrm{C} 40$

$\mathrm{C} 38-\mathrm{C} 37-\mathrm{C} 40$

$\mathrm{C} 33-\mathrm{C} 38-\mathrm{C} 37$

C33-C $38-\mathrm{H} 38$

C $37-\mathrm{C} 38-\mathrm{H} 38$

$\mathrm{O} 5-\mathrm{C} 39-\mathrm{O} 6$

$\mathrm{O} 5-\mathrm{C} 39-\mathrm{C} 35$

$\mathrm{O} 6-\mathrm{C} 39-\mathrm{C} 35$

$\mathrm{C} 39-\mathrm{O} 5-\mathrm{Zn} 2$

$\mathrm{C} 39-\mathrm{O} 6-\mathrm{Zn} 1$

O7- $440-08$

$\mathrm{O} 7-\mathrm{C} 40-\mathrm{C} 37$

$\mathrm{O} 8-\mathrm{C} 40-\mathrm{C} 37$

$\mathrm{C} 40-\mathrm{O} 7-\mathrm{Zn}^{\mathrm{iv}}$

$\mathrm{C} 40-\mathrm{O} 8-\mathrm{Zn} 1^{\mathrm{iv}}$

$\mathrm{C} 43-\mathrm{N} 3-\mathrm{C} 41$

$\mathrm{C} 43-\mathrm{N} 3-\mathrm{C} 42$

$\mathrm{C} 41-\mathrm{N} 3-\mathrm{C} 42$

N3-C41-H41A

N3-C41-H41B
119.1 (2)

120.4

120.4

$121.2(2)$

119.4

119.4

121.3 (2)

119.4

119.4

116.9 (2)

121.5

121.5

128.81 (18)

$122.6(2)$

108.6 (2)

$120.68(17)$

$120.16(17)$

$119.16(17)$

119.69 (17)

120.2

120.2

120.11 (17)

119.62 (17)

$120.21(16)$

119.81 (16)

120.1

120.1

120.32 (16)

119.59 (16)

$120.05(16)$

119.33 (17)

120.3

120.3

$127.29(17)$

116.57 (16)

116.14 (16)

$133.92(12)$

134.38 (12)

125.74 (17)

$117.86(16)$

$116.36(15)$

$128.02(12)$

121.14 (12)

123.3 (2)

120.3 (2)

116.3 (2)

109.5

109.5 


\begin{tabular}{|c|c|}
\hline $\mathrm{C} 8-\mathrm{C} 9-\mathrm{H} 9$ & 119.4 \\
\hline $\mathrm{C} 10-\mathrm{C} 9-\mathrm{H} 9$ & 119.4 \\
\hline $\mathrm{C} 11-\mathrm{C} 10-\mathrm{C} 9$ & $120.9(2)$ \\
\hline $\mathrm{C} 11-\mathrm{C} 10-\mathrm{H} 10$ & 119.6 \\
\hline $\mathrm{C} 9-\mathrm{C} 10-\mathrm{H} 10$ & 119.6 \\
\hline $\mathrm{C} 12-\mathrm{C} 11-\mathrm{C} 10$ & $117.7(2)$ \\
\hline $\mathrm{C} 12-\mathrm{C} 11-\mathrm{H} 11$ & 121.2 \\
\hline $\mathrm{C} 10-\mathrm{C} 11-\mathrm{H} 11$ & 121.2 \\
\hline $\mathrm{C} 11-\mathrm{C} 12-\mathrm{N} 1$ & $129.34(18)$ \\
\hline $\mathrm{C} 11-\mathrm{C} 12-\mathrm{C} 7$ & $121.91(19)$ \\
\hline $\mathrm{N} 1-\mathrm{C} 12-\mathrm{C} 7$ & $108.75(18)$ \\
\hline $\mathrm{C} 18-\mathrm{C} 13-\mathrm{C} 14$ & $120.81(16)$ \\
\hline $\mathrm{C} 18-\mathrm{C} 13-\mathrm{N} 1$ & $118.69(17)$ \\
\hline $\mathrm{C} 14-\mathrm{C} 13-\mathrm{N} 1$ & $120.50(18)$ \\
\hline $\mathrm{C} 13-\mathrm{C} 14-\mathrm{C} 15$ & $118.82(18)$ \\
\hline $\mathrm{C} 13-\mathrm{C} 14-\mathrm{H} 14$ & 120.6 \\
\hline $\mathrm{C} 15-\mathrm{C} 14-\mathrm{H} 14$ & 120.6 \\
\hline $\mathrm{C} 16-\mathrm{C} 15-\mathrm{C} 14$ & $120.63(18)$ \\
\hline $\mathrm{C} 16-\mathrm{C} 15-\mathrm{C} 19$ & $119.67(17)$ \\
\hline $\mathrm{C} 14-\mathrm{C} 15-\mathrm{C} 19$ & $119.64(17)$ \\
\hline $\mathrm{C} 17-\mathrm{C} 16-\mathrm{C} 15$ & $120.06(17)$ \\
\hline $\mathrm{C} 17-\mathrm{C} 16-\mathrm{H} 16$ & 120.0 \\
\hline $\mathrm{C} 15-\mathrm{C} 16-\mathrm{H} 16$ & 120.0 \\
\hline $\mathrm{C} 16-\mathrm{C} 17-\mathrm{C} 18$ & $119.76(18)$ \\
\hline $\mathrm{C} 16-\mathrm{C} 17-\mathrm{C} 20$ & $120.68(16)$ \\
\hline $\mathrm{C} 18-\mathrm{C} 17-\mathrm{C} 20$ & $119.55(17)$ \\
\hline $\mathrm{C} 13-\mathrm{C} 18-\mathrm{C} 17$ & $119.91(17)$ \\
\hline $\mathrm{C} 13-\mathrm{C} 18-\mathrm{H} 18$ & 120.0 \\
\hline $\mathrm{C} 17-\mathrm{C} 18-\mathrm{H} 18$ & 120.0 \\
\hline $\mathrm{O} 2-\mathrm{C} 19-\mathrm{O} 1$ & $124.52(17)$ \\
\hline $\mathrm{O} 2-\mathrm{C} 19-\mathrm{C} 15$ & $117.67(17)$ \\
\hline $\mathrm{O} 1-\mathrm{C} 19-\mathrm{C} 15$ & $117.81(16)$ \\
\hline $\mathrm{C} 19-\mathrm{O} 1-\mathrm{Zn} 1^{\mathrm{iii}}$ & $108.90(12)$ \\
\hline $\mathrm{C} 19-\mathrm{O} 2-\mathrm{Zn} 2^{\mathrm{iii}}$ & $168.46(13)$ \\
\hline $\mathrm{O} 3-\mathrm{C} 20-\mathrm{O} 4$ & $124.97(17)$ \\
\hline $\mathrm{O} 3-\mathrm{C} 20-\mathrm{C} 17$ & $119.93(17)$ \\
\hline $\mathrm{O} 4-\mathrm{C} 20-\mathrm{C} 17$ & $115.10(16)$ \\
\hline $\mathrm{C} 20-\mathrm{O} 4-\mathrm{Zn} 1$ & $108.79(12)$ \\
\hline $\mathrm{C} 32-\mathrm{N} 2-\mathrm{C} 21$ & $108.95(16)$ \\
\hline $\mathrm{C} 32-\mathrm{N} 2-\mathrm{C} 33$ & $125.96(17)$ \\
\hline $\mathrm{C} 21-\mathrm{N} 2-\mathrm{C} 33$ & $124.11(18)$ \\
\hline $\mathrm{C} 22-\mathrm{C} 21-\mathrm{N} 2$ & $128.86(18)$ \\
\hline $\mathrm{C} 22-\mathrm{C} 21-\mathrm{C} 26$ & $122.47(19)$ \\
\hline $\mathrm{N} 2-\mathrm{C} 21-\mathrm{C} 26$ & $108.58(19)$ \\
\hline $\mathrm{C} 23-\mathrm{C} 22-\mathrm{C} 21$ & $117.5(2)$ \\
\hline $\mathrm{C} 23-\mathrm{C} 22-\mathrm{H} 22$ & 121.2 \\
\hline $\mathrm{C} 21-\mathrm{C} 22-\mathrm{H} 22$ & 121.2 \\
\hline $\mathrm{C} 22-\mathrm{C} 23-\mathrm{C} 24$ & $121.3(2)$ \\
\hline
\end{tabular}

$\begin{array}{ll}\text { H41A-C41-H41B } & 109.5 \\ \text { N3-C41-H41C } & 109.5 \\ \text { H41A-C41-H41C } & 109.5 \\ \text { H41B-C41-H41C } & 109.5 \\ \text { N3-C42-H42A } & 109.5 \\ \text { N3-C42-H42B } & 109.5 \\ \text { H42A-C42-H42B } & 109.5 \\ \text { N3-C42-H42C } & 109.5 \\ \text { H42A-C42-H42C } & 109.5 \\ \text { H42B-C42-H42C } & 109.5 \\ \text { O9-C43-N3 } & 124.0(2) \\ \text { O9-C43-H43 } & 118.0 \\ \text { N3-C43-H43 } & 118.0 \\ \text { C43-O9-Zn2 } & 121.99(15) \\ \text { C46-N4-C44 } & 120.20(18) \\ \text { C46-N4-C45 } & 121.41(17) \\ \text { C44-N4-C45 } & 118.25(17) \\ \text { N4-C44-H44A } & 109.5 \\ \text { N4-C44-H44B } & 109.5 \\ \text { H44A-C44-H44B } & 109.5 \\ \text { N4-C44-H44C } & 109.5 \\ \text { H44A-C44-H44C } & 109.5 \\ \text { H44B-C44-H44C } & 109.5 \\ \text { N4-C45-H45A } & 109.5 \\ \text { N4-C45-H45B } & 109.5 \\ \text { H45A-C45-H45B } & 109.5 \\ \text { N4-C45-H45C } & 109.5 \\ \text { H45A-C45-H45C } & 109.5 \\ \text { H45B-C45-H45C } & 109.5 \\ \text { O10-C46-N4 } & 124.13(19) \\ \text { O10-C46-H46 } & 117.9 \\ \text { N4-C46-H46 } & 117.9 \\ \text { C46-O10-Zn2 } & 116.49(12) \\ \text { C49-N5-C47 } & 121.42(19) \\ \text { C49-N5-C48 } & 120.73(19) \\ \text { C47-N5-C48 } & 117.77(18) \\ \text { N5-C47-H47A } & 109.5 \\ \text { N5-C47-H47B } & 109.5 \\ \text { H47A-C47-H47B } & 109.5 \\ \text { N5-C47-H47C } & 109.5 \\ \text { H47A-C47-H47C } & 109.5 \\ \text { H47B-C47-H47C } & 109.5 \\ \text { N5-C48-H48A } & 109.5 \\ \text { N5-C48-H48B } & 109.5 \\ \text { H48A-C48-H48B } & \\ \text { N5-C48-H48C } & \text { H48A-C48-H48C } \\ \text { H48B-C48-H48C } & \\ & \end{array}$




\begin{tabular}{|c|c|c|c|}
\hline $\mathrm{C} 22-\mathrm{C} 23-\mathrm{H} 23$ & 119.3 & $\mathrm{O} 11-\mathrm{C} 49-\mathrm{N} 5$ & $125.0(2)$ \\
\hline $\mathrm{C} 24-\mathrm{C} 23-\mathrm{H} 23$ & 119.3 & $\mathrm{O} 11-\mathrm{C} 49-\mathrm{H} 49$ & 117.5 \\
\hline $\mathrm{C} 25-\mathrm{C} 24-\mathrm{C} 23$ & $120.7(2)$ & $\mathrm{N} 5-\mathrm{C} 49-\mathrm{H} 49$ & 117.5 \\
\hline $\mathrm{C} 25-\mathrm{C} 24-\mathrm{H} 24$ & 119.7 & $\mathrm{O} 12-\mathrm{C} 50-\mathrm{H} 50 \mathrm{~A}$ & 109.5 \\
\hline $\mathrm{C} 23-\mathrm{C} 24-\mathrm{H} 24$ & 119.7 & $\mathrm{O} 12-\mathrm{C} 50-\mathrm{H} 50 \mathrm{~B}$ & 109.5 \\
\hline $\mathrm{C} 24-\mathrm{C} 25-\mathrm{C} 26$ & $119.8(2)$ & $\mathrm{H} 50 \mathrm{~A}-\mathrm{C} 50-\mathrm{H} 50 \mathrm{~B}$ & 109.5 \\
\hline $\mathrm{C} 24-\mathrm{C} 25-\mathrm{H} 25$ & 120.1 & $\mathrm{O} 12-\mathrm{C} 50-\mathrm{H} 50 \mathrm{C}$ & 109.5 \\
\hline $\mathrm{C} 26-\mathrm{C} 25-\mathrm{H} 25$ & 120.1 & $\mathrm{H} 50 \mathrm{~A}-\mathrm{C} 50-\mathrm{H} 50 \mathrm{C}$ & 109.5 \\
\hline $\mathrm{C} 25-\mathrm{C} 26-\mathrm{C} 21$ & $118.1(2)$ & $\mathrm{H} 50 \mathrm{~B}-\mathrm{C} 50-\mathrm{H} 50 \mathrm{C}$ & 109.5 \\
\hline $\mathrm{C} 25-\mathrm{C} 26-\mathrm{C} 27$ & $135.0(2)$ & $\mathrm{C} 50-\mathrm{O} 12-\mathrm{Zn} 2$ & $128.06(13)$ \\
\hline $\mathrm{C} 21-\mathrm{C} 26-\mathrm{C} 27$ & $106.75(18)$ & $\mathrm{C} 50-\mathrm{O} 12-\mathrm{H} 12 \mathrm{O}$ & 107.3 \\
\hline $\mathrm{C} 28-\mathrm{C} 27-\mathrm{C} 32$ & $118.9(2)$ & $\mathrm{Zn} 2-\mathrm{O} 12-\mathrm{H} 12 \mathrm{O}$ & 124.2 \\
\hline $\mathrm{C} 28-\mathrm{C} 27-\mathrm{C} 26$ & $134.0(2)$ & & \\
\hline $\mathrm{C} 12-\mathrm{N} 1-\mathrm{C} 1-\mathrm{C} 2$ & $-178.7(2)$ & $\mathrm{C} 26-\mathrm{C} 21-\mathrm{C} 22-\mathrm{C} 23$ & $-1.8(3)$ \\
\hline $\mathrm{C} 13-\mathrm{N} 1-\mathrm{C} 1-\mathrm{C} 2$ & $13.6(3)$ & $\mathrm{C} 21-\mathrm{C} 22-\mathrm{C} 23-\mathrm{C} 24$ & $1.0(3)$ \\
\hline $\mathrm{C} 12-\mathrm{N} 1-\mathrm{C} 1-\mathrm{C} 6$ & $-1.0(2)$ & $\mathrm{C} 22-\mathrm{C} 23-\mathrm{C} 24-\mathrm{C} 25$ & $0.8(3)$ \\
\hline $\mathrm{C} 13-\mathrm{N} 1-\mathrm{C} 1-\mathrm{C} 6$ & $-168.66(18)$ & $\mathrm{C} 23-\mathrm{C} 24-\mathrm{C} 25-\mathrm{C} 26$ & $-1.8(3)$ \\
\hline $\mathrm{N} 1-\mathrm{C} 1-\mathrm{C} 2-\mathrm{C} 3$ & $177.2(2)$ & $\mathrm{C} 24-\mathrm{C} 25-\mathrm{C} 26-\mathrm{C} 21$ & $1.0(3)$ \\
\hline $\mathrm{C} 6-\mathrm{C} 1-\mathrm{C} 2-\mathrm{C} 3$ & $-0.4(3)$ & $\mathrm{C} 24-\mathrm{C} 25-\mathrm{C} 26-\mathrm{C} 27$ & $-173.6(2)$ \\
\hline $\mathrm{C} 1-\mathrm{C} 2-\mathrm{C} 3-\mathrm{C} 4$ & $-0.8(3)$ & $\mathrm{C} 22-\mathrm{C} 21-\mathrm{C} 26-\mathrm{C} 25$ & $0.8(3)$ \\
\hline $\mathrm{C} 2-\mathrm{C} 3-\mathrm{C} 4-\mathrm{C} 5$ & $1.0(4)$ & $\mathrm{N} 2-\mathrm{C} 21-\mathrm{C} 26-\mathrm{C} 25$ & $-175.98(17)$ \\
\hline $\mathrm{C} 3-\mathrm{C} 4-\mathrm{C} 5-\mathrm{C} 6$ & $0.0(4)$ & $\mathrm{C} 22-\mathrm{C} 21-\mathrm{C} 26-\mathrm{C} 27$ & $176.83(18)$ \\
\hline $\mathrm{C} 4-\mathrm{C} 5-\mathrm{C} 6-\mathrm{C} 1$ & $-1.2(3)$ & $\mathrm{N} 2-\mathrm{C} 21-\mathrm{C} 26-\mathrm{C} 27$ & $0.0(2)$ \\
\hline $\mathrm{C} 4-\mathrm{C} 5-\mathrm{C} 6-\mathrm{C} 7$ & $-179.4(2)$ & $\mathrm{C} 25-\mathrm{C} 26-\mathrm{C} 27-\mathrm{C} 28$ & $-6.7(4)$ \\
\hline $\mathrm{N} 1-\mathrm{C} 1-\mathrm{C} 6-\mathrm{C} 5$ & $-176.59(19)$ & $\mathrm{C} 21-\mathrm{C} 26-\mathrm{C} 27-\mathrm{C} 28$ & $178.2(2)$ \\
\hline $\mathrm{C} 2-\mathrm{C} 1-\mathrm{C} 6-\mathrm{C} 5$ & $1.4(3)$ & $\mathrm{C} 25-\mathrm{C} 26-\mathrm{C} 27-\mathrm{C} 32$ & $173.3(2)$ \\
\hline $\mathrm{N} 1-\mathrm{C} 1-\mathrm{C} 6-\mathrm{C} 7$ & $2.1(2)$ & $\mathrm{C} 21-\mathrm{C} 26-\mathrm{C} 27-\mathrm{C} 32$ & $-1.7(2)$ \\
\hline $\mathrm{C} 2-\mathrm{C} 1-\mathrm{C} 6-\mathrm{C} 7$ & $-179.99(18)$ & $\mathrm{C} 32-\mathrm{C} 27-\mathrm{C} 28-\mathrm{C} 29$ & $-0.9(3)$ \\
\hline $\mathrm{C} 5-\mathrm{C} 6-\mathrm{C} 7-\mathrm{C} 8$ & $-4.4(4)$ & $\mathrm{C} 26-\mathrm{C} 27-\mathrm{C} 28-\mathrm{C} 29$ & $179.2(2)$ \\
\hline $\mathrm{C} 1-\mathrm{C} 6-\mathrm{C} 7-\mathrm{C} 8$ & $177.2(2)$ & $\mathrm{C} 27-\mathrm{C} 28-\mathrm{C} 29-\mathrm{C} 30$ & $0.1(3)$ \\
\hline $\mathrm{C} 5-\mathrm{C} 6-\mathrm{C} 7-\mathrm{C} 12$ & $176.0(2)$ & $\mathrm{C} 28-\mathrm{C} 29-\mathrm{C} 30-\mathrm{C} 31$ & $0.4(4)$ \\
\hline $\mathrm{C} 1-\mathrm{C} 6-\mathrm{C} 7-\mathrm{C} 12$ & $-2.4(2)$ & $\mathrm{C} 29-\mathrm{C} 30-\mathrm{C} 31-\mathrm{C} 32$ & $-0.1(3)$ \\
\hline $\mathrm{C} 12-\mathrm{C} 7-\mathrm{C} 8-\mathrm{C} 9$ & $-1.1(3)$ & $\mathrm{C} 30-\mathrm{C} 31-\mathrm{C} 32-\mathrm{N} 2$ & $177.3(2)$ \\
\hline $\mathrm{C} 6-\mathrm{C} 7-\mathrm{C} 8-\mathrm{C} 9$ & $179.4(2)$ & $\mathrm{C} 30-\mathrm{C} 31-\mathrm{C} 32-\mathrm{C} 27$ & $-0.7(3)$ \\
\hline $\mathrm{C} 7-\mathrm{C} 8-\mathrm{C} 9-\mathrm{C} 10$ & $-0.7(3)$ & $\mathrm{C} 21-\mathrm{N} 2-\mathrm{C} 32-\mathrm{C} 31$ & $179.0(2)$ \\
\hline $\mathrm{C} 8-\mathrm{C} 9-\mathrm{C} 10-\mathrm{C} 11$ & $1.0(3)$ & $\mathrm{C} 33-\mathrm{N} 2-\mathrm{C} 32-\mathrm{C} 31$ & $10.0(3)$ \\
\hline $\mathrm{C} 9-\mathrm{C} 10-\mathrm{C} 11-\mathrm{C} 12$ & $0.5(3)$ & $\mathrm{C} 21-\mathrm{N} 2-\mathrm{C} 32-\mathrm{C} 27$ & $-2.8(2)$ \\
\hline $\mathrm{C} 10-\mathrm{C} 11-\mathrm{C} 12-\mathrm{N} 1$ & $178.3(2)$ & $\mathrm{C} 33-\mathrm{N} 2-\mathrm{C} 32-\mathrm{C} 27$ & $-171.78(18)$ \\
\hline $\mathrm{C} 10-\mathrm{C} 11-\mathrm{C} 12-\mathrm{C} 7$ & $-2.3(3)$ & $\mathrm{C} 28-\mathrm{C} 27-\mathrm{C} 32-\mathrm{C} 31$ & $1.2(3)$ \\
\hline $\mathrm{C} 1-\mathrm{N} 1-\mathrm{C} 12-\mathrm{C} 11$ & $178.9(2)$ & $\mathrm{C} 26-\mathrm{C} 27-\mathrm{C} 32-\mathrm{C} 31$ & $-178.88(19)$ \\
\hline $\mathrm{C} 13-\mathrm{N} 1-\mathrm{C} 12-\mathrm{C} 11$ & $-13.4(3)$ & $\mathrm{C} 28-\mathrm{C} 27-\mathrm{C} 32-\mathrm{N} 2$ & $-177.18(18)$ \\
\hline $\mathrm{C} 1-\mathrm{N} 1-\mathrm{C} 12-\mathrm{C} 7$ & $-0.6(2)$ & $\mathrm{C} 26-\mathrm{C} 27-\mathrm{C} 32-\mathrm{N} 2$ & $2.8(2)$ \\
\hline $\mathrm{C} 13-\mathrm{N} 1-\mathrm{C} 12-\mathrm{C} 7$ & $167.07(18)$ & $\mathrm{C} 32-\mathrm{N} 2-\mathrm{C} 33-\mathrm{C} 38$ & $-117.0(2)$ \\
\hline $\mathrm{C} 8-\mathrm{C} 7-\mathrm{C} 12-\mathrm{C} 11$ & $2.6(3)$ & $\mathrm{C} 21-\mathrm{N} 2-\mathrm{C} 33-\mathrm{C} 38$ & $75.7(3)$ \\
\hline $\mathrm{C} 6-\mathrm{C} 7-\mathrm{C} 12-\mathrm{C} 11$ & $-177.73(19)$ & $\mathrm{C} 32-\mathrm{N} 2-\mathrm{C} 33-\mathrm{C} 34$ & $63.0(3)$ \\
\hline $\mathrm{C} 8-\mathrm{C} 7-\mathrm{C} 12-\mathrm{N} 1$ & $-177.81(18)$ & $\mathrm{C} 21-\mathrm{N} 2-\mathrm{C} 33-\mathrm{C} 34$ & $-104.4(2)$ \\
\hline $\mathrm{C} 6-\mathrm{C} 7-\mathrm{C} 12-\mathrm{N} 1$ & $1.8(2)$ & $\mathrm{C} 38-\mathrm{C} 33-\mathrm{C} 34-\mathrm{C} 35$ & $-2.4(3)$ \\
\hline
\end{tabular}




\begin{tabular}{|c|c|c|c|}
\hline $\mathrm{C} 1-\mathrm{N} 1-\mathrm{C} 13-\mathrm{C} 18$ & $57.1(3)$ & $\mathrm{N} 2-\mathrm{C} 33-\mathrm{C} 34-\mathrm{C} 35$ & $177.66(18)$ \\
\hline $\mathrm{C} 12-\mathrm{N} 1-\mathrm{C} 13-\mathrm{C} 18$ & $-108.6(2)$ & $\mathrm{C} 33-\mathrm{C} 34-\mathrm{C} 35-\mathrm{C} 36$ & $1.1(3)$ \\
\hline $\mathrm{C} 1-\mathrm{N} 1-\mathrm{C} 13-\mathrm{C} 14$ & $-123.2(2)$ & $\mathrm{C} 33-\mathrm{C} 34-\mathrm{C} 35-\mathrm{C} 39$ & $178.31(17)$ \\
\hline $\mathrm{C} 12-\mathrm{N} 1-\mathrm{C} 13-\mathrm{C} 14$ & $71.1(3)$ & $\mathrm{C} 34-\mathrm{C} 35-\mathrm{C} 36-\mathrm{C} 37$ & $1.2(3)$ \\
\hline $\mathrm{C} 18-\mathrm{C} 13-\mathrm{C} 14-\mathrm{C} 15$ & $-1.1(3)$ & $\mathrm{C} 39-\mathrm{C} 35-\mathrm{C} 36-\mathrm{C} 37$ & $-175.98(17)$ \\
\hline $\mathrm{N} 1-\mathrm{C} 13-\mathrm{C} 14-\mathrm{C} 15$ & $179.23(17)$ & $\mathrm{C} 35-\mathrm{C} 36-\mathrm{C} 37-\mathrm{C} 38$ & $-2.3(3)$ \\
\hline $\mathrm{C} 13-\mathrm{C} 14-\mathrm{C} 15-\mathrm{C} 16$ & $0.3(3)$ & $\mathrm{C} 35-\mathrm{C} 36-\mathrm{C} 37-\mathrm{C} 40$ & $175.25(17)$ \\
\hline $\mathrm{C} 13-\mathrm{C} 14-\mathrm{C} 15-\mathrm{C} 19$ & $-176.94(17)$ & $\mathrm{C} 34-\mathrm{C} 33-\mathrm{C} 38-\mathrm{C} 37$ & $1.2(3)$ \\
\hline $\mathrm{C} 14-\mathrm{C} 15-\mathrm{C} 16-\mathrm{C} 17$ & $0.9(3)$ & $\mathrm{N} 2-\mathrm{C} 33-\mathrm{C} 38-\mathrm{C} 37$ & $-178.79(18)$ \\
\hline $\mathrm{C} 19-\mathrm{C} 15-\mathrm{C} 16-\mathrm{C} 17$ & $178.11(17)$ & $\mathrm{C} 36-\mathrm{C} 37-\mathrm{C} 38-\mathrm{C} 33$ & $1.1(3)$ \\
\hline $\mathrm{C} 15-\mathrm{C} 16-\mathrm{C} 17-\mathrm{C} 18$ & $-1.2(3)$ & $\mathrm{C} 40-\mathrm{C} 37-\mathrm{C} 38-\mathrm{C} 33$ & $-176.43(17)$ \\
\hline $\mathrm{C} 15-\mathrm{C} 16-\mathrm{C} 17-\mathrm{C} 20$ & $179.98(17)$ & $\mathrm{C} 34-\mathrm{C} 35-\mathrm{C} 39-\mathrm{O} 5$ & $-14.1(3)$ \\
\hline $\mathrm{C} 14-\mathrm{C} 13-\mathrm{C} 18-\mathrm{C} 17$ & $0.7(3)$ & $\mathrm{C} 36-\mathrm{C} 35-\mathrm{C} 39-\mathrm{O} 5$ & $163.01(18)$ \\
\hline $\mathrm{N} 1-\mathrm{C} 13-\mathrm{C} 18-\mathrm{C} 17$ & $-179.58(17)$ & $\mathrm{C} 34-\mathrm{C} 35-\mathrm{C} 39-\mathrm{O} 6$ & $166.52(18)$ \\
\hline $\mathrm{C} 16-\mathrm{C} 17-\mathrm{C} 18-\mathrm{C} 13$ & $0.4(3)$ & $\mathrm{C} 36-\mathrm{C} 35-\mathrm{C} 39-\mathrm{O} 6$ & $-16.3(3)$ \\
\hline $\mathrm{C} 20-\mathrm{C} 17-\mathrm{C} 18-\mathrm{C} 13$ & $179.24(17)$ & $\mathrm{O} 6-\mathrm{C} 39-\mathrm{O} 5-\mathrm{Zn} 2$ & $28.8(3)$ \\
\hline $\mathrm{C} 16-\mathrm{C} 15-\mathrm{C} 19-\mathrm{O} 2$ & $-10.1(3)$ & $\mathrm{C} 35-\mathrm{C} 39-\mathrm{O} 5-\mathrm{Zn} 2$ & $-150.43(14)$ \\
\hline $\mathrm{C} 14-\mathrm{C} 15-\mathrm{C} 19-\mathrm{O} 2$ & $167.10(17)$ & $\mathrm{O} 5-\mathrm{C} 39-\mathrm{O} 6-\mathrm{Zn} 1$ & $-6.2(3)$ \\
\hline $\mathrm{C} 16-\mathrm{C} 15-\mathrm{C} 19-\mathrm{O} 1$ & $170.02(17)$ & $\mathrm{C} 35-\mathrm{C} 39-\mathrm{O} 6-\mathrm{Zn} 1$ & $173.02(12)$ \\
\hline $\mathrm{C} 14-\mathrm{C} 15-\mathrm{C} 19-\mathrm{O} 1$ & $-12.7(3)$ & $\mathrm{C} 36-\mathrm{C} 37-\mathrm{C} 40-\mathrm{O} 7$ & $5.3(3)$ \\
\hline $\mathrm{O} 2-\mathrm{C} 19-\mathrm{O} 1-\mathrm{Zn} 1^{\mathrm{iii}}$ & $-3.8(2)$ & $\mathrm{C} 38-\mathrm{C} 37-\mathrm{C} 40-\mathrm{O} 7$ & $-177.10(17)$ \\
\hline $\mathrm{C} 15-\mathrm{C} 19-\mathrm{O} 1-\mathrm{Zn} 1^{\mathrm{iii}}$ & $175.98(13)$ & $\mathrm{C} 36-\mathrm{C} 37-\mathrm{C} 40-\mathrm{O} 8$ & $-172.62(17)$ \\
\hline $\mathrm{O} 1-\mathrm{C} 19-\mathrm{O} 2-\mathrm{Zn} 2^{\mathrm{iii}}$ & $3.9(8)$ & $\mathrm{C} 38-\mathrm{C} 37-\mathrm{C} 40-\mathrm{O} 8$ & $4.9(3)$ \\
\hline $\mathrm{C} 15-\mathrm{C} 19-\mathrm{O} 2-\mathrm{Zn} 2^{\mathrm{iii}}$ & $-175.9(6)$ & $\mathrm{O} 8-\mathrm{C} 40-\mathrm{O} 7-\mathrm{Zn} 2^{\mathrm{iv}}$ & $-59.3(3)$ \\
\hline $\mathrm{C} 16-\mathrm{C} 17-\mathrm{C} 20-\mathrm{O} 3$ & $11.8(3)$ & $\mathrm{C} 37-\mathrm{C} 40-\mathrm{O} 7-\mathrm{Zn} 2^{\mathrm{iv}}$ & $122.99(16)$ \\
\hline $\mathrm{C} 18-\mathrm{C} 17-\mathrm{C} 20-\mathrm{O} 3$ & $-166.93(17)$ & $\mathrm{O} 7-\mathrm{C} 40-\mathrm{O} 8-\mathrm{Zn} 1^{\mathrm{iv}}$ & $-8.6(3)$ \\
\hline $\mathrm{C} 16-\mathrm{C} 17-\mathrm{C} 20-\mathrm{O} 4$ & $-167.72(17)$ & $\mathrm{C} 37-\mathrm{C} 40-\mathrm{O} 8-\mathrm{Zn} 1^{\mathrm{iv}}$ & $169.16(12)$ \\
\hline $\mathrm{C} 18-\mathrm{C} 17-\mathrm{C} 20-\mathrm{O} 4$ & $13.5(2)$ & $\mathrm{C} 41-\mathrm{N} 3-\mathrm{C} 43-\mathrm{O} 9$ & $-6.4(4)$ \\
\hline $\mathrm{O} 3-\mathrm{C} 20-\mathrm{O} 4-\mathrm{Zn} 1$ & $5.5(2)$ & $\mathrm{C} 42-\mathrm{N} 3-\mathrm{C} 43-\mathrm{O} 9$ & $178.5(2)$ \\
\hline $\mathrm{C} 17-\mathrm{C} 20-\mathrm{O} 4-\mathrm{Zn} 1$ & $-174.97(12)$ & $\mathrm{N} 3-\mathrm{C} 43-\mathrm{O} 9-\mathrm{Zn} 2$ & $149.88(19)$ \\
\hline $\mathrm{C} 32-\mathrm{N} 2-\mathrm{C} 21-\mathrm{C} 22$ & $-174.83(19)$ & $\mathrm{C} 44-\mathrm{N} 4-\mathrm{C} 46-\mathrm{O} 10$ & $-0.8(3)$ \\
\hline $\mathrm{C} 33-\mathrm{N} 2-\mathrm{C} 21-\mathrm{C} 22$ & $-5.6(3)$ & $\mathrm{C} 45-\mathrm{N} 4-\mathrm{C} 46-\mathrm{O} 10$ & $174.8(2)$ \\
\hline $\mathrm{C} 32-\mathrm{N} 2-\mathrm{C} 21-\mathrm{C} 26$ & $1.7(2)$ & $\mathrm{N} 4-\mathrm{C} 46-\mathrm{O} 10-\mathrm{Zn} 2$ & $-150.09(18)$ \\
\hline $\mathrm{C} 33-\mathrm{N} 2-\mathrm{C} 21-\mathrm{C} 26$ & $170.93(17)$ & $\mathrm{C} 47-\mathrm{N} 5-\mathrm{C} 49-\mathrm{O} 11$ & $-1.9(3)$ \\
\hline $\mathrm{N} 2-\mathrm{C} 21-\mathrm{C} 22-\mathrm{C} 23$ & $174.29(19)$ & $\mathrm{C} 48-\mathrm{N} 5-\mathrm{C} 49-\mathrm{O} 11$ & $-178.5(2)$ \\
\hline
\end{tabular}

Symmetry codes: (i) $-x+1, y+1 / 2,-z+3 / 2$; (ii) $x-1, y, z$; (iii) $x+1, y, z$; (iv) $-x+1, y-1 / 2,-z+3 / 2$.

Hydrogen-bond geometry $\left(A,{ }^{\circ}\right)$

$\mathrm{Cg} 1, \mathrm{Cg} 3, \mathrm{Cg} 4$ and $\mathrm{Cg} 6$ are the centroids of the $\mathrm{N} 1 / \mathrm{C} 1 / \mathrm{C} 6 / \mathrm{C} 7 / \mathrm{C} 12, \mathrm{C} 1-\mathrm{C} 6, \mathrm{C} 7-\mathrm{C} 12$ and $\mathrm{C} 21-\mathrm{C} 26$ rings, respectively.

\begin{tabular}{lllll}
\hline$D-\mathrm{H} \cdots A$ & $D-\mathrm{H}$ & $\mathrm{H} \cdots A$ & $D \cdots A$ & $D-\mathrm{H} \cdots A$ \\
\hline $\mathrm{O} 12-\mathrm{H} 12 O \cdots \mathrm{O} 11$ & 0.76 & 1.93 & $2.696(2)$ & 174 \\
$\mathrm{C} 42-\mathrm{H} 42 B \cdots \mathrm{O} 11^{\mathrm{v}}$ & 0.98 & 2.52 & $3.327(3)$ & 139 \\
$\mathrm{C} 43-\mathrm{H} 43 \cdots \mathrm{O} 10$ & 0.95 & 2.44 & $3.028(3)$ & 120 \\
$\mathrm{C} 46-\mathrm{H} 46 \cdots \mathrm{O} 5$ & 0.95 & 2.39 & $2.995(2)$ & 121 \\
$\mathrm{C} 47-\mathrm{H} 47 B^{\cdots} \mathrm{O} 3^{\text {iv }}$ & 0.98 & 2.61 & $3.417(3)$ & 140 \\
$\mathrm{C} 47-\mathrm{H} 47 C \cdots 9^{\text {vi }}$ & 0.98 & 2.65 & $3.608(3)$ & 165
\end{tabular}


supporting information

\begin{tabular}{lllll}
$\mathrm{C} 2-\mathrm{H} 2 \cdots C g 6^{\text {vii }}$ & 0.95 & 2.64 & $3.517(2)$ & 153 \\
$\mathrm{C} 10-\mathrm{H} 10 \cdots C g 3^{\text {viii }}$ & 0.95 & 2.64 & $3.418(2)$ & 139 \\
$\mathrm{C} 44-\mathrm{H} 44 B \cdots C g 4^{\text {ix }}$ & 0.98 & 2.91 & $3.596(2)$ & 128 \\
$\mathrm{C} 45-\mathrm{H} 45 B \cdots C g 1^{\text {ix }}$ & 0.98 & 2.85 & $3.411(2)$ & 117 \\
\hline
\end{tabular}

Symmetry codes: (iv) $-x+1, y-1 / 2,-z+3 / 2$; (v) $-x, y+1 / 2,-z+3 / 2$; (vi) $-x, y-1 / 2,-z+3 / 2$; (vii) $-x+3 / 2,-y+2, z-1 / 2$; (viii) $x+1 / 2,-y+3 / 2,-z+1$; (ix) $-x+3 / 2,-y+2, z+1 / 2$. 